\title{
Asymptotics of the Susceptibility for the Ising Model on the Hierarchical Lattices
}

\author{
P. M. Bleher ${ }^{1}$ and E. Žalys ${ }^{2}$ \\ ${ }^{1}$ Institute of Applied Mathematics, Academy of Sciences of the USSR, SU-125047 Moscow, \\ USSR \\ ${ }^{2}$ Institute of Mathematics and Cybernetics, Academy of Sciences of the Lithuanian SSR, \\ Akademijos 4, SU-232600 Vilnius, USSR
}

\begin{abstract}
Using the GKS inequality Griffiths and Kaufman showed in [12] that the susceptibility in the Ising model on the hierarchical lattices is infinite for $T>T_{c}$. In the present work we give an analytic calculation of the dominant singularity of the susceptibility when $h \rightarrow 0$ for $T>T_{c}$. Moreover we analytically find the spontaneous magnetization for $T<T_{c}$ and prove that for $T<T_{c}$ the susceptibility is finite.
\end{abstract}

\section{Introduction}

In the present paper the Ising model on the "diamond hierarchical lattices" (DHL) is considered. The models of such type were introduced first by Migdal [1] and Kadanoff [2] in the context of an approximate real space renormalization group (RG) scheme for the Ising model on $Z^{d}$. In the following papers of Berker and Ostlund [3] and Bleher and Žalys [4] it was shown that the Migdal-Kadanoff RG equations become exact for the models on DHL. In the fundamental paper of Griffiths and Kaufman [5] general definitions, examples and main properties of the hierarchical lattices (HL) were given and discussed (the term "HL" was introduced first in [5]). After that in many papers (see [6-13]) various spin models on the HL were studied as independent (from any approximate RG scheme) objects of the investigation. In our previous paper [14] we analysed (following [5]) the thermodynamical limit of the ferromagnetic Ising model on the HL and proved that the limit Gibbs state is unique unless $T<T_{c}, h=0$, and in that case the number of pure Gibbs states is equal to 2 ( + and - phases).

An intriguing result was obtained in [12] where it was shown that in the Ising model on the HL the susceptibility is infinite for $T>T_{c}$. The main idea of the paper [12] was a calculation of the (dimensionless) susceptibility at $T=\infty$ (noninteracting spins) which turned out to be infinite. Then the required result was obtained using the GKS inequality which states the magnetization increases with decreasing of $T$.

Here, we calculate analytically the dominant singularity of the susceptibility when $h \rightarrow 0$ for $T>T_{c}$. It turns out to be logarithmic in the two-dimensional case 
and powerlike if the dimension $d>2$. Detailed formulae are given below. For $T=\infty$ our results coincide with those of Kaufman and Griffiths [12]. For $T<T_{c}$ we find an explicit formula for the spontaneous magnetization and show that the susceptibility is finite and even infinitely smooth at $h=0$ (when $h \rightarrow+0$ or -0 ). As was noted in [12] the singularity of the susceptibility for $T>T_{c}$ is connected with an unusual behaviour of the zeroes of the partition function of the Ising model on the HL. Further (in Sect. 4) we obtain a simple equations for the zeroes of the partition function and present some results of their distributions for different values of temperature.

The set-up of this paper is the following. In Sect. 2 we recall the definition of the Ising model on the HL and analyse the system of the RG equations for the model. In Sect. 3 we present our main results concerning the analytical calculations of the spontaneous magnetization and the magnetic susceptibility. The distribution of the zeroes of the partition function, as mentioned above, is discussed in Sect. 4. All detailed proofs of the main statements of the paper are given in Appendices $A, B$; an algorithm of the zeroes of the partition function computation is given in Appendix $C$.

\section{The Ising model on the DHL}

The diamond hierarchical lattice which is used later on is generated iteratively with the help of the following procedure (see [5]). Let $\Gamma$ be a graph consisting of two "external" vertices $\alpha, \tau$, and $b \geqq 1$ "internal" ones and $2 b$ edges which connect $\alpha$ and $\tau$ with the internal vertices (see Fig. 1). $\Gamma$ is called the generating graph for the DHL. Let $\Gamma_{0}$ be the zero-order DHL which consists of two external (or "boundary") vertices $\alpha_{0}, \tau_{0}$ and a single edge connecting these vertices. To obtain lattice $\Gamma_{n+1}$ from $\Gamma_{n}$ one has to replace each edge of $\Gamma_{n}$ by the graph $\Gamma$ identifying the vertices $\alpha$ and $\tau$ with the endpoints of the edge (see Fig. 1). The images $\alpha_{n+1}, \tau_{n+1}$ of the external vertices $\alpha_{n}, \tau_{n}$ in this replacement are the external vertices of $\Gamma_{n+1}$. Examples of more general HL arising by using the arbitrary connected generating graph $\Gamma$ with two external (marked) vertices are given in [5]. The number $b \geqq 1$ is a parameter of the DHL. The number $B=2 b$ is called the aggregation number of the DHL (see [5]) and $d=1+(\log b) / \log 2$ is the "dimensiality" of the DHL because the $d$-dimensional Migdal-Kadanoff RG equations are exact for the spin models on the DHL with $b=2^{d-1}$.

Fig. 1

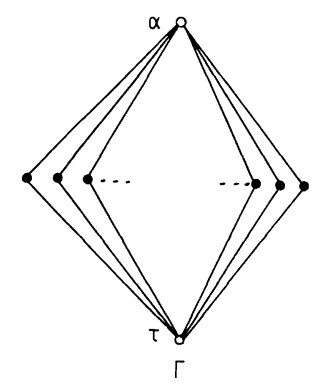

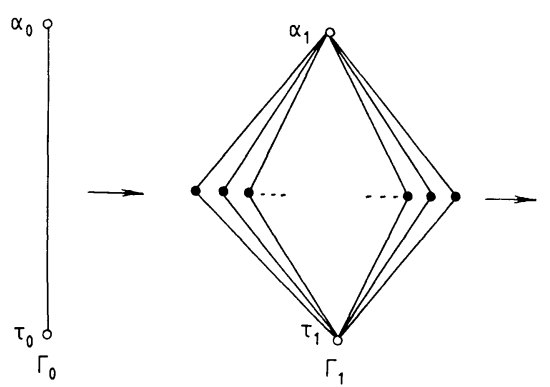


The Ising model on the DHL is defined in the usual way by associating spin variables $\sigma_{i}= \pm 1$ with the vertices $i \in \Gamma_{n}$ and by setting the Hamiltonian

$$
H_{n}\left(\sigma^{n}\right)=-\frac{J}{2} \sum_{\langle i, j\rangle \in \Gamma_{n}} \sigma_{i} \sigma_{j}-\frac{h}{2} \sum_{\langle i, j\rangle \in \Gamma_{n}}\left(\sigma_{i}+\sigma_{j}\right),
$$

where the sums go over all pairs $\langle i, j\rangle$ of the nearest neighbours in $\Gamma_{n}$ (two vertices $i$, $j$ are the nearest neighbours iff they are connected by an edge), $J$ is the exchange interaction, $h$ is the external field.

The iterative procedure of the DHL construction leads to recurrent equations for restricted (conditional) partition functions which enable us to obtain analytic representations for the free energy, the spontaneous magnetization and other thermodynamical functions. Define the restricted partition function by

$$
Z_{n}\left(\sigma_{\alpha_{n}}, \sigma_{\tau_{n}}\right)=\sum_{\sigma^{n} \mid \sigma_{\alpha_{n}}, \sigma_{\tau_{n}}} \exp \left\{\frac{1}{k T} H_{n}\left(\sigma^{n}\right)\right\},
$$

where the sum goes over all the configurations $\sigma^{n}=\left\{\sigma_{i}, i \in \Gamma_{n}\right\}$ with fixed values of the external spins $\sigma_{\alpha_{n}}, \sigma_{\tau_{n}}$. Further we assume the Boltzman factor $k=1$. Denote:

$$
P_{n}=Z_{n}(+1,+1) ; \quad Q_{n}=Z_{n}(+1,-1)=Z_{n}(-1,+1) ; \quad R_{n}=Z_{n}(-1,-1) .
$$

Then the following recurrent equations are valid:

$$
P_{n+1}=\left(P_{n}^{2}+Q_{n}^{2}\right)^{b} ; \quad Q_{n+1}=\left(P_{n}+R_{n}\right)^{b} Q_{n}^{b} ; \quad R_{n+1}=\left(R_{n}^{2}+Q_{n}^{2}\right)^{b} .
$$

To prove them first one has to take the sum over all internal spins of the sublattices $\Gamma_{n}$ forming the lattice $\Gamma_{n+1}$, and next take the sum over all external spins of these sublattices with fixed values of $\sigma_{\alpha_{n+1}}, \sigma_{\tau_{n+1}}$. So we get the Eq. (1). Note that they are just the Migdal-Kadanoff RG equations.

Equations (1) are supplied with the initial conditions

$$
P_{0}=\exp \left(\frac{J}{2 T}+\frac{h}{T}\right) ; \quad Q_{0}=\exp \left(-\frac{J}{2 T}\right) ; \quad R_{0}=\exp \left(\frac{J}{2 T}-\frac{h}{T}\right) .
$$

It is convenient to introduce relative variables,

$$
z_{n}=\left(R_{n} / P_{n}\right)^{1 / b} ; \quad t_{n}=\left(Q_{n}^{2} / P_{n} R_{n}\right)^{1 / b},
$$

which will be called the RG variables. From (1) the RG recurrent equations for them follow:

$$
z_{n}=\frac{z_{n}^{b}+t_{n}^{b}}{z_{n}^{-b}+t_{n}^{b}} ; \quad t_{n}=\frac{z_{n}^{b}+z_{n}^{-b}+2}{z_{n}^{b}+z_{n}^{-b}+t_{n}^{b}+t_{n}^{-b}},
$$

with the initial conditions

$$
z_{0}=\exp \left(-\frac{2 h}{b T}\right) ; \quad t_{0}=\exp \left(-\frac{2 J}{b T}\right) .
$$

The initial conditions motivate the representation

$$
z_{n}=\exp \left(-\frac{2 h_{n}}{b T_{n}}\right) ; \quad t_{n}=\exp \left(-\frac{2 J}{b T_{n}}\right)
$$


where $T_{n}$ and $h_{n}$ are called the renormalized temperature and external field, respectively.

Now we are going to discuss some properties of the RG equations (3). Further we shall assume $b>1$ everywhere, because there is no phase transition for $b=1$.

1. If $z_{n}=1$, then $z_{n+1}=1$ and

$$
t_{n+1}=4 /\left(2+t_{n}^{b}+t_{n}^{-b}\right) \equiv f\left(t_{n}\right),
$$

which means that the line $z_{n} \equiv 1$ corresponding to the Ising model with $h=0$ is invariant with respect to the $R G$ equations (3). Equation (5) has a unique fixed point $t_{c}$ in the interval $(0,1)$ for which

$$
t_{c}=4 /\left(2+t_{c}^{b}+t_{c}^{-b}\right) .
$$

(For a rigorous proof of this and the following statements see the appendices at the end of the paper.) The fixed point $t_{c}$ is unstable and $\lim t_{n}=0$ if $0<t_{0}<t_{c}$ and $\lim _{n \rightarrow \infty} t_{n}=1$ if $t_{c}<t_{0}<1$. The points $t=0$ and $t=1$ are the low- and high-temperature fixed points of the RG equation (5), respectively. They are stable and even superstable fixed points. Indeed, from (5) it easily follows that $f^{\prime}(0)=f^{\prime \prime}(0)$ $=\ldots=f^{(b-1)}(0), f^{\prime}(1)=0$, so

$$
\begin{gathered}
0<t_{n+1}<C t_{n}^{b}, \\
0<1-t_{n+1}<C\left(1-t_{n}\right)^{2},
\end{gathered}
$$

if $0<t_{n}<1$ with an appropriate constant $C>0$. As a consequence of this the superexponential convergences $t_{n} \leqq C_{1} \exp \left(-C_{2} b^{n}\right)$, when $t_{n} \rightarrow 0$ and $1-t_{n}$ $\leqq C_{1} \exp \left(-C_{2} 2^{n}\right)$, when $t_{n} \rightarrow 1$ hold. Here and below by $C, C_{0}, C_{1}, \ldots$ we denote various factors which do not depend on $n$. They can be different even in close but different formulae. Usually we point out the variables on which these factors depend.

The equation $t_{c}=\exp \left(-2 J / b T_{c}\right)$ (following from (4)) determines the critical temperature $T_{c}=T_{c}(b)$. In Table 1 the values of the reduced critical temperature $T_{c} / J$ are given for some values of $b$.

2. From (3) one can easily verify that $\left\{0<z_{n+1}<1,0<t_{n+1}<1\right\}$ if $\left\{0<z_{n}<1\right.$, $\left.0<t_{n}<1\right\}$, which means that the square $Q^{2}=(0,1) \times(0,1)$ is invariant with respect to the RG equations (3). By continuity one can extend the transformation $\mathscr{R}:\left\{z_{n}, t_{n}\right\} \rightarrow\left\{z_{n+1}, t_{n+1}\right\}$ determined by Eq. (3) to the boundary of $Q^{2}$ everywhere except the point $\{0,0\}$. Note that from (3) it follows that $z_{n+1} \leqq z_{n}^{b}$, so all the fixed points of the RG transformation $\mathscr{R}$ lie on the lines $z \equiv 0$ and $z \equiv 1$. As $\mathscr{R}:\{0, t\}$

Table 1

\begin{tabular}{llllllll}
\hline$b$ & 2 & 3 & 4 & 5 & 10 & 50 & 100 \\
\hline$T_{c} / J$ & 0.8205 & 1.3854 & 1.915 & 2.4326 & 4.9665 & 24.993 & 49.9999 \\
\hline
\end{tabular}


Fig. 2

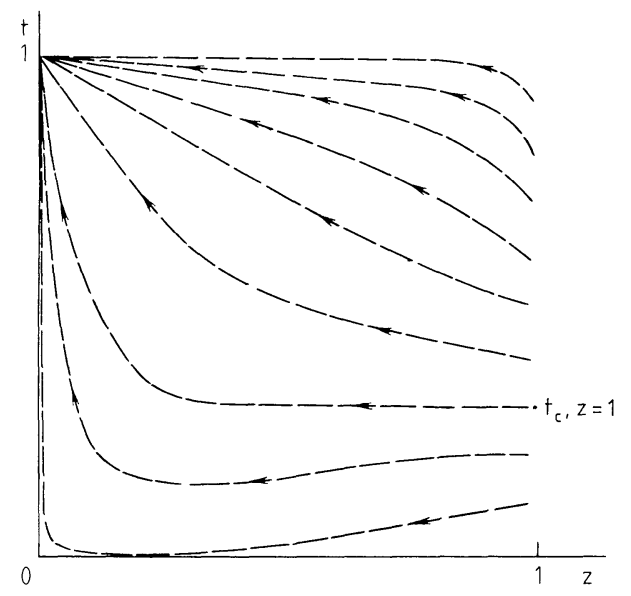

$\rightarrow\{0,1\}$ for $t \neq 0$, the only fixed point on the line $z=0$ is $\{0,1\}$. This fixed point of the RG transformation corresponds to the positive external field. It is superstable and all trajectories starting inside $Q^{2}$ tend to it. Some of these trajectories are shown in Fig. 2.

3. The RG transformation $\mathscr{R}:\left\{z_{n}, t_{n}\right\} \rightarrow\left\{z_{n+1}, t_{n+1}\right\}$ commutes with the transformation $S: z \rightarrow \frac{1}{z}$. This property is connected obviously with the invariance of the Hamiltonian of the Ising model with respect to the transformation $\sigma \rightarrow-\sigma$, $h \rightarrow-h$. It enables us to prove the following equalities by induction in $n$ :

$$
z_{n}(T,-h)=z_{n}^{-1}(T, h) ; \quad t_{n}(T,-h)=t_{n}(T, h) .
$$

The latter one leads to the equality

$$
\left.\frac{\partial t_{n}}{\partial h}\right|_{h=0}=0
$$

Moreover due to (6),

$$
\begin{aligned}
\left.\frac{\partial z_{n}}{\partial h}\right|_{h=0}= & \left.\left.\frac{\partial z_{n}}{\partial z_{n-1}}\right|_{z_{n-1}=1} \cdot \frac{\partial z_{n-1}}{\partial h}\right|_{h=0}+\left.\frac{\partial z_{n}}{\partial t_{n-1}}\right|_{z_{n-1}=1} \\
& \times\left.\frac{\partial t_{n-1}}{\partial h}\right|_{h=0}=\left.\left.\frac{\partial z_{n}}{\partial z_{n-1}}\right|_{z_{n-1}=1} \cdot \frac{\partial z_{n-1}}{\partial h}\right|_{h=0}
\end{aligned}
$$

As $\left.\frac{\partial z_{n}}{\partial z_{n-1}}\right|_{z_{n-1}=1}=\frac{2 b}{1+t_{n-1}^{b}}$, then

$$
\left.\frac{\partial z_{n}}{\partial h}\right|_{h=0}=\left.\frac{2 b}{1+t_{n-1}^{b}} \cdot \frac{\partial z_{n-1}}{\partial h}\right|_{h=0} .
$$


Using this equation recurrently we have

$$
\left.\frac{\partial z_{n}}{\partial h}\right|_{h=0}=-\frac{2}{b T} \prod_{j=0}^{n-1} \frac{2 b}{1+t_{j}^{b}} .
$$

The formulae (6) and (7) obtained here will be used below for a direct calculation of the thermodynamical functions.

\section{Thermodynamical Functions}

The free energy of the Ising model on the DHL can be found in terms of the RG variables $\left\{z_{n}, t_{n}\right\}$ [see (2)]. We start with Eq. (1) which gives

$$
P_{n+1}=P_{n}^{2 b}\left(1+\left(Q_{n} / P_{n}\right)^{2}\right)^{b}=P_{n}^{2 b}\left(1+z_{n}^{b} t_{n}^{b}\right)^{b} .
$$

Using this equality recurrently we have

$$
\begin{aligned}
P_{n}= & P_{n-1}^{2 b}\left(1+z_{n-1}^{b} t_{n-1}^{b}\right)^{b}=P_{n-1}^{(2 b)^{2}}\left(1+z_{n-2}^{b} t_{n-2}^{b}\right)^{b \cdot 2 b} \\
& \times\left(1+z_{n-1}^{b} t_{n-1}^{b}\right)^{b}=\ldots=P_{0}^{(2 b)^{n}} \prod_{j=0}^{n-1}\left(1+z_{j}^{b} t_{j}^{b}\right)^{b(2 b)^{n-1-j}} \\
= & \exp \left\{(2 b)^{n}\left(\frac{J}{2 T}+\frac{h}{T}\right)\right\} \prod_{j=0}^{n-1}\left(1+z_{j}^{b} t_{j}^{b}\right)^{b(2 b)^{n-1-j}} .
\end{aligned}
$$

Let

$$
\Xi_{n}=\sum_{\sigma^{n}} \exp \left\{-T^{-1} H_{n}\left(\sigma^{n}\right)\right\}
$$

be the grand partition function. As

$$
\Xi_{n}=P_{n}+2 Q_{n}+R_{n}=P_{n}\left(1+2 Q_{n} / P_{n}+R_{n} / P_{n}\right)=P_{n}\left(1+2 z_{n}^{b / 2} t_{n}^{b / 2}+z_{n}^{b}\right),
$$

we obtain

$$
\Xi_{n}=\exp \left\{(2 b)^{n}\left(\frac{J}{2 T}+\frac{h}{T}\right)\right\}\left(1+2 z_{n}^{b / 2} t_{n}^{b / 2}+z_{n}^{b}\right) \prod_{j=0}^{n-1}\left(1+z_{j}^{b} t_{j}^{b}\right)^{b(2 b)^{n-1-j}} .
$$

In order to get a right relation between the free energy and the magnetization in the Ising model on the DHL one must take into account the total number of the terms $-h \sigma_{i}, i \in \Gamma_{n}$ in $H_{n}\left(\sigma^{n}\right)$ which is equal to $(2 b)^{n}$, so the free energy $F=-T \lim _{n \rightarrow \infty}(2 b)^{-n} \ln \Xi_{n}$, and due to (9)

$$
\begin{aligned}
F= & -\frac{J}{2}-h-\frac{T}{2} \lim _{n \rightarrow \infty} \sum_{j=0}^{n-1}(2 b)^{-j} \ln \left(1+z_{j}^{b} t_{j}^{b}\right) \\
& -\lim _{n \rightarrow \infty}(2 b)^{-n} \ln \left(1+2 z_{n}^{b / 2} t_{n}^{b / 2}+z_{n}^{b}\right) .
\end{aligned}
$$

Assume $0<z_{0} \leqq 1$ (which means $h \geqq 0$ ) and $0<t_{0}<1$. Then $0<z_{n} \leqq 1$ and $0<t_{n}<1$, so the series $\sum_{n=0}^{\infty}(2 b)^{-n} \ln \left(1+z_{n}^{b} t_{n}^{b}\right)$ converges uniformly and

$$
\lim _{n \rightarrow \infty}(2 b)^{-n} \ln \left(1+2 z_{n}^{b / 2} t_{n}^{b / 2}+z_{n}^{b}\right)=0 .
$$


Hence it follows that

$$
F=-\frac{J}{2}-h-\frac{T}{2} \sum_{n=0}^{\infty}(2 b)^{-n} \ln \left(1+z_{n}^{b} t_{n}^{b}\right)
$$

It is not difficult to verify that this formula remains valid for $h<0$ as well, but we shall use it only for $h \geqq 0$.

Another formula for free energy can be obtained similarly when one starts with another variant of relation (8):

$$
\Xi_{n}=Q_{n}\left(\frac{P_{n}}{Q_{n}}+2+\frac{R_{n}}{Q_{n}}\right)=Q_{n}\left(z_{n}^{-b / 2} t_{n}^{-b / 2}+2+z_{n}^{b / 2} t_{n}^{-b / 2}\right),
$$

and uses the recurrent equation for $Q_{n}$. In this case we get

$$
F=-\frac{J}{2}-\frac{T}{2} \sum_{n=0}^{\infty}(2 b)^{-n} \ln \left\{t_{n}^{-b / 2}\left(z_{n}^{-b / 2}+z_{n}^{b / 2}\right)\right\} .
$$

To calculate the spontaneous magnetization we differentiate both sides of Eq. (10) in $h$ and put $h \rightarrow+0$. Using relations (6), (7) we have

$$
\begin{aligned}
M & =-\left.\frac{\partial F}{\partial h}\right|_{h=+0}=1+\left.\frac{T}{2} \frac{\partial}{\partial h}\left\{\sum_{n=0}^{\infty}(2 b)^{-n} \ln \left(1+z_{n}^{b} t_{n}^{b}\right)\right\}\right|_{h=+0} \\
& =1+\left.\frac{T}{2} \sum_{n=0}^{\infty}(2 b)^{-n} \frac{b t_{n}^{b}}{1+t_{n}^{b}} \cdot \frac{\partial z_{n}}{\partial h}\right|_{h=+0} \\
& =1+\frac{T}{2} \sum_{n=0}^{\infty}(2 b)^{-n} \frac{b t_{n}^{b}}{1+t_{n}^{b}}\left(-\frac{2}{b T} \prod_{j=0}^{n-1} \frac{2 b}{1+t_{j}^{b}}\right) \\
& =1-\sum_{n=0}^{\infty}\left[\prod_{j=0}^{n-1}\left(1+t_{j}^{b}\right)^{-1}-\prod_{j=0}^{n}\left(1+t_{j}^{b}\right)^{-1}\right] .
\end{aligned}
$$

Thus

$$
M(t)=\prod_{n=0}^{\infty}\left(1+t_{n}^{b}\right)^{-1},
$$

where $t_{n+1}=4 \cdot\left(2+t_{n}^{b}+t_{n}^{-b}\right)^{-1}, t_{0}=\exp (-2 J / b T)$. It is noteworthy that these calculations of $M(T)$ must be done very carefully. For instance, similar differentiating of formula (11) would lead to $M(T)=0$. The problem is in the uniform differentiability in $h$ of the series in formulae (10), (11). As is proved in the appendices the series in (10) is uniformly differentiable in $h$ for $h \geqq 0$, while that in (11) is not.

Consider formula (12) more closely. As $\lim _{n \rightarrow 0} t_{n}=1$ for $T>T_{c}, M(T)=0$ in this case. Similarly, $M\left(T_{c}\right)=0$ as $t_{n}=t_{c}, n \geqq 0$. Moreover, $M(T)>0$ for $T<T_{c}$ and $\lim _{T \rightarrow 0} M(T)=1, M(T)=C(T)\left(T_{c}-T\right)^{\beta}$ as $T \rightarrow T_{c}-0$, where $C(T)$ is an oscillating factor and

$$
\beta=\frac{\log \left(1+t_{c}^{b}\right)}{\log f^{\prime}\left(t_{c}\right)}
$$


Table 2

\begin{tabular}{llllllll}
\hline$b$ & 2 & 3 & 4 & 5 & 10 & 50 & 100 \\
\hline$\beta$ & 0.161734 & 0.343271 & 0.463242 & 0.546752 & 0.745762 & 0.943172 & 0.970517 \\
\hline
\end{tabular}

is the critical exponent. To see it let us note that by (12)

$$
M\left(t_{1}\right)=\left(1+t_{0}^{b}\right) M\left(t_{0}\right) .
$$

(This formula can be considered as the "RG equation" for the spontaneous magnetization.) If $t_{0}=t_{c}-\varepsilon, \varepsilon \downarrow 0$, then

$$
t_{1}=f\left(t_{0}\right) \approx t_{c}-f^{\prime}\left(t_{c}\right) \varepsilon \text { and } \quad\left(1+t_{0}^{b}\right) \approx\left(1+t_{c}^{b}\right),
$$

so

$$
M\left(t_{c}-f^{\prime}\left(t_{c}\right) \varepsilon\right) \approx\left(1+t_{c}^{b}\right) M\left(t_{c}-\varepsilon\right) .
$$

Let

$$
M\left(t_{c}-\varepsilon\right)=C(\varepsilon) \varepsilon^{\beta} .
$$

Then

$$
C\left(f^{\prime}\left(t_{c}\right) \varepsilon\right)\left[f^{\prime}\left(t_{c}\right) \varepsilon\right]^{\beta} \approx\left(1+t_{c}^{b}\right) \varepsilon^{\beta} C(\varepsilon)
$$

or

$$
C\left(f^{\prime}\left(t_{c}\right) \varepsilon\right) \approx C(\varepsilon)\left[\left(1+t_{c}^{b}\right)\left(f^{\prime}\left(t_{c}\right)\right)^{-\beta}\right] .
$$

For $\beta$ determined by (13), $\left(1+t_{c}^{b}\right)\left(f^{\prime}\left(t_{c}\right)\right)^{-\beta}=1$, and hence $C\left(f^{\prime}\left(t_{c}\right) \varepsilon\right) \approx C(\varepsilon)$. As $f^{\prime}\left(t_{c}\right)>1$, the last equation means that $C(\varepsilon)$ is oscillating periodically when $\varepsilon \downarrow 0$. As $M\left(t_{c}-\varepsilon\right)$ is positive for $\varepsilon>0$, one can show that $C_{2}>C(\varepsilon)>C_{1}>0$.

In Table 2 the values of the critical exponent $\beta$ are given for some values of $b$.

Note for comparison that $\beta=1 / 8=0.125$ in the Ising model on $Z^{2}$ and $\beta \approx 5 / 16$ $=0.3125$ in the Ising model on $Z^{3}$. The corresponding values in Table 2 are those for $b=2$ and 4 (as $b=2^{d-1}$ ). One can see that the agreement is better for $d=2$ than for $d=3$. This reflects the general tendency that the Migdal-Kadanoff approximation works better near the low critical dimension which in our case is equal to 1 . Moreover, it is exact in the first order of the perturbation theory in the dimension $d=1+\varepsilon$ (see [1]).

The divergence of the "dimensionless" susceptibility $\hat{\chi}=-T^{2}\left(\partial^{2} F / \partial h^{2}\right)$ was shown at $T=\infty$ by a direct computation in [12]. By the GKS inequality it implies the divergence of the susceptibility (more precisely of the quantity $h^{-1} M(T, h)$, where $M$ is the magnetization) for any $T>T_{c}$. We calculate the dominant singularity of the susceptibility for $T>T_{c}$ in the following

Proposition 1. Let $\chi=-\frac{\partial^{2} F}{\partial h^{2}}, T>T_{c}, b=2^{d-1}$. Then

$$
\chi(T, h)=\left\{\begin{array}{l}
\frac{A^{2}(T)}{2 T}\left[\log _{2} \frac{1}{|h|}+2 K\left(\frac{2 A(T)}{T}|h|\right)\right]+r(T, h), \quad \text { if } \quad b=2, \\
\frac{A^{2}(T)}{T}\left[\frac{2 A(T)}{T}|h|\right]^{-\gamma} K\left(\frac{2 A(T)}{T}|h|\right)+r(T, h), \quad \text { if } \quad b>2,
\end{array}\right.
$$


where

$$
\begin{gathered}
A(T)=\prod_{n=0}^{\infty}\left[2\left(1+t_{n}^{-b}\right)^{-1}\right]>0, \\
t_{n+1}=4 /\left(2+t_{n}^{b}+t_{n}^{-b}\right), \quad t_{0}=\exp (-2 J / b T), \\
\gamma=1-\log _{2}^{-1} b,
\end{gathered}
$$

and $\lim _{h \rightarrow+0} r(T, h)=r(T, 0)$ exists, $|r(T, 0)|<\infty$. For any argument $x>0$ function $K(x)$ has the following properties:
a) $K(b x)=K(x)$,
b) $K(x)$ is analytic in $x$,
c) $K(x)>0$, if $b>2$.

Function $K(x)$ is bounded and periodic with respect to the variable $\log _{b}|x|$. The appearance of such oscillating factors is typical for various asymptotics of thermodynamical functions of the spin models on the DHL (see [10]).

Proof of Proposition 1 is given in Appendix A. The following statement is proved in Appendix B.

Proposition 2. For any $T<T_{c}$ the susceptibility $\chi(T, 0)=\lim _{h \rightarrow+0} \chi(T, h)$ is finite.

\section{Zeroes of the Partition Function}

The divergence of the susceptibility at $h=0$ implies that the point $h=0$ is a limit point of the set of zeroes of the partition function

$$
\Xi_{n}=\sum_{\sigma^{n}} \exp \left\{-\frac{1}{T} H_{n}\left(h, \sigma^{n}\right)\right\}
$$

for any fixed $T$. In this section we discuss some properties of the location of zeroes of the partition function $\Xi_{n}$. To avoid some non-essential technical difficulties we shall assume that $b$ is even.

According to the Lee-Yang theorem (see e.g. [15]) all zeroes of the partition function $\Xi_{n}$ lie on the imaginary axis in $h$. If $h$ is imaginary, then $\left|z_{0}\right|$ $=|\exp (-2 h / b T)|=1$. Let $\Sigma_{n}$ be the set of zeroes of the partition function $\Xi_{n}$ $=\Xi_{n}\left(z_{0}, t_{0}\right)$ in the cylinder

$$
Y=\left\{z_{0} \in \mathbb{C},\left|z_{0}\right|=1\right\} \times\left\{0 \leqq t_{0}<1\right\},
$$

where

$$
z_{0}=\exp (-2 h / b T) ; \quad t_{0}=\exp (-2 J / b T) .
$$

Note first of all that for any fixed $T$ (i.e. fixed $t_{0}$ ),

$$
\Xi_{n}=\sum_{k=0}^{2(2 b)^{n}} m_{k}(T) \exp \left\{\frac{h}{T}\left[(2 b)^{n}-k\right]\right\},
$$


where

$$
\begin{aligned}
& m_{k}(T)=\sum_{\sigma \in S_{k}}\left[\exp (J / 2 T) \sum_{\langle i, j\rangle} \sigma_{i} \sigma_{j}\right], \\
& S_{k}=\left\{\sigma \mid \frac{1}{2} \sum_{\langle i, j\rangle}\left(\sigma_{i}+\sigma_{j}\right)=(2 b)^{n}-k\right\},
\end{aligned}
$$

so

$$
\begin{aligned}
\Xi_{n} & =\exp \left\{(2 b)^{n} \frac{h}{T}\right\} \sum_{k=0}^{2(2 b)^{n}} m_{k}(T) \exp (-k h / T) \\
& =z_{0}^{-b(2 b)^{n} / 2} \sum_{k=0}^{2(2 b)^{n}} m_{k}(T) z_{0}^{b k / 2},
\end{aligned}
$$

therefore $\Xi_{n} z_{0}^{b(2 b)^{n} / 2}$ is a polynomial in $z_{0}$ of the $b(2 b)^{n}$ order and $\Xi_{n}$ has $b(2 b)^{n}$ zeroes.

RG equations (3) have the following property: if $\left|z_{n}\right|=1, z_{n}=\exp \left(i \varphi_{n}\right)$ and $0 \leqq t_{n}<1$, then

$$
\begin{gathered}
\left|z_{n+1}\right|=\left|\frac{\exp \left(i b \varphi_{n}\right)+t_{n}^{b}}{\exp \left(-i b \varphi_{n}\right)+t_{n}^{b}}\right|=1, \\
0 \leqq t_{n+1}=\frac{\cos \left(b \varphi_{n}\right)+1}{\cos \left(b \varphi_{n}\right)+\left(t_{n}^{b}+t_{n}^{-b}\right) / 2}<1 .
\end{gathered}
$$

In other words, the $\mathrm{RG}$ transformation $\mathscr{R}$ maps the cylinder $Y$ into itself. Moreover, note that in formula (9) for $\Xi_{n}$ the only factor $\left(1+2 z_{n}^{b / 2} t_{n}^{b / 2}+z_{n}^{b}\right)$ can vanish for imaginary $h$. Indeed, as $\left(z_{j}, t_{j}\right) \in Y, j=0,1, \ldots, n$, so $\left|z_{j}^{b} t_{j}^{b}\right|=t_{j}^{b}<1$, and hence $1+z_{j}^{b} t_{j}^{b} \neq 0$. The equation

$$
0=1+2 z_{n}^{b / 2} t_{n}^{b / 2}+z_{n}^{b}=2 z_{n}^{b / 2}\left[t_{n}^{b / 2}+\left(z_{n}^{b / 2}+z_{n}^{-b / 2}\right) / 2\right]
$$

leads to

$$
t_{n}^{b / 2}+\left(z_{n}^{b / 2}+z_{n}^{-b / 2}\right) / 2=0
$$

or $\cos \left(b \varphi_{n} / 2\right)+t_{n}^{b / 2}=0$. This equation determines the location of the zeroes of the partition function $\Xi_{n}$. It is useful for us to reformulate it in the following recurrent equation for the sets $\Sigma_{n}$ :

$$
\begin{gathered}
\Sigma_{n+1}=\mathscr{R}^{-1}\left(\Sigma_{n}\right), \\
\Sigma_{0}=\left\{\left(z_{0}, t_{0}\right) \in Y \mid t_{0}^{b / 2}+\left(z_{0}^{b / 2}+z_{0}^{-b / 2}\right) / 2=0\right\},
\end{gathered}
$$

where $\mathscr{R}^{-1}(X)$ denotes the full pre-image of a set $X \subset Y$ with respect to $\mathscr{R}$, i.e. $\mathscr{R}^{-1}(X)=\{y \mid \mathscr{R} y \in X\}$.

To describe the sets $\Sigma_{n}$ consider some properties of the transformation $\mathscr{R}$. Let

$$
Y_{0}=\left\{(z, t) \in Y \mid z=\exp (i \varphi), 0<\varphi<\frac{\pi}{b}, 0<t<1\right\} .
$$

We call $Y_{0}$ the fundamental domain for $\mathscr{R} . \mathscr{R}$ maps the boundary of $Y_{0}$ in the following way:

1. $\{\varphi=0,0 \leqq t \leqq 1\} \rightarrow\{\varphi=0,0 \leqq t \leqq 1\}$,

2. $\{0 \leqq \varphi \leqq \pi / b, t=0\} \rightarrow\{0 \leqq \varphi<2 \pi, t=0\}, \varphi \rightarrow 2 b \varphi$, 
Fig. 3

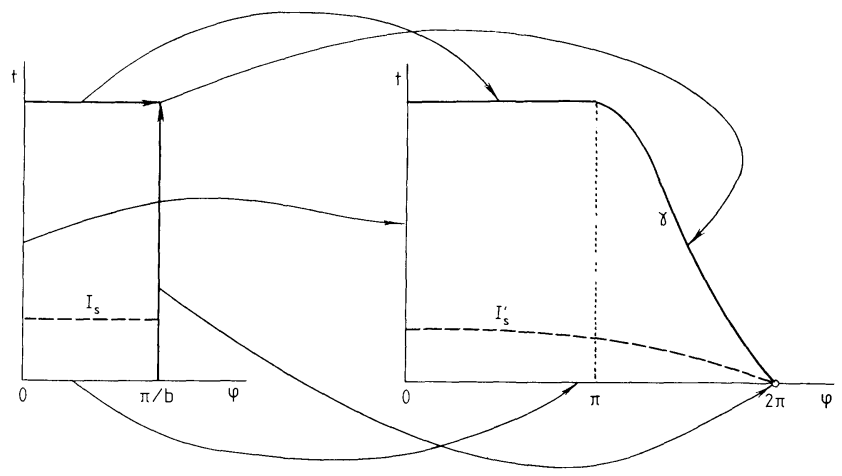

3. $\{0 \leqq \varphi<\pi / b, t=1\} \rightarrow\{0 \leqq \varphi<\pi, t=1\}, \varphi \rightarrow b \varphi$,

4. $\{\varphi=\pi / b, 0 \leqq t<1\} \rightarrow\{\varphi=2 \pi, t=0\}$

(see Fig. 3). For the point $\{\varphi=\pi / b, t=1\}$ the map $\mathscr{R}$ is not defined. Let us show that this point "is mapped" to the curve

$$
\gamma=\left\{(z, t) \mid z=\exp (i \varphi), t=\sin ^{2}(\varphi / 2), \pi \leqq \varphi \leqq 2 \pi\right\} .
$$

It means that curve $\gamma$ is the set of limit points of $\mathscr{R} y$ when $y \rightarrow(\exp (\pi i / b), 1), y \in Y_{0}$. Let $t=1-\varepsilon / b, \varphi=(\pi-\omega \varepsilon) / b$, where $\omega>0$ is fixed and $\varepsilon>0$ goes to 0 . Then calculations in the major order in $\varepsilon$ give $t^{b} \approx 1-\varepsilon$,

$$
\begin{aligned}
z^{b}= & \exp (\pi i-\omega \varepsilon i)=-\exp (-\omega \varepsilon i)=-\cos (\omega \varepsilon) \\
& +i \sin (\omega \varepsilon) \approx-1+(\omega \varepsilon)^{2} / 2+i \omega \varepsilon,
\end{aligned}
$$

so

$$
z^{\prime}=\frac{z^{b}+t^{b}}{z^{-b}+t^{b}} \approx \frac{(\omega \varepsilon)^{2} / 2-\varepsilon+i \omega \varepsilon}{(\omega \varepsilon)^{2} / 2-\varepsilon-i \omega \varepsilon} \approx \frac{1-i \omega}{1+i \omega}=\exp \left(i \varphi^{\prime}\right)
$$

where $\varphi^{\prime}=-2 \operatorname{arctg} \omega$ or $\omega=\operatorname{tg}\left(-\varphi^{\prime} / 2\right)$. Next,

$$
\begin{aligned}
t^{\prime} & =\frac{z^{b}+z^{-b}+2}{z^{b}+z^{-b}+t^{b}+t^{-b}} \approx \frac{(\omega \varepsilon)^{2}}{(\omega \varepsilon)^{2}+\varepsilon^{2}}=\frac{\operatorname{tg}^{2}\left(-\varphi^{\prime} / 2\right)}{\operatorname{tg}^{2}\left(-\varphi^{\prime} / 2\right)+1} \\
& =\sin ^{2}\left(-\varphi^{\prime} / 2\right)=\sin ^{2}\left(\varphi^{\prime} / 2\right) .
\end{aligned}
$$

Thus $\varphi^{\prime}=-2 \operatorname{arctg} \omega, \omega>0$ varies from $-\pi$ to 0 , or from $\pi$ to $2 \pi$ and $t^{\prime}=\sin ^{2}\left(\varphi^{\prime} / 2\right)$ as was stated.

Consider the open domain $Y_{1} \subset Y$ enclosed with the curve

$$
\{0 \leqq \varphi<2 \pi, t=0\} \cup \gamma \cup\{0 \leqq \varphi<\pi, t=1\} \cup\{\varphi=0,0 \leqq t \leqq 1\}
$$

(see Fig. 3). $\mathscr{R}$ maps $Y_{0}$ onto $Y_{1}$ diffeomorphically. Each segment $I_{s}=\{0 \leqq \varphi \leqq \pi / b$, $t=s, 0 \leqq s<1\}$ stretches to curve $I_{s}^{\prime}=\left\{0 \leqq \varphi^{\prime} \leqq 2 \pi, t^{\prime}=v_{s}\left(\varphi^{\prime}\right), v_{s}(2 \pi)=0\right\}$ (see Fig. 3) and $\partial \varphi^{\prime} / \partial \varphi \geqq 2 b\left(1+t_{n}^{b}\right)^{-1} \geqq b$.

Let us return now to Eq. (16). For simplicity assume that $b=2$. For $n=0, \Sigma_{0}$ $=\{\cos \varphi+\mathrm{t}=0\}$ (see Fig. 4). Turn to $\Sigma_{1}=\mathscr{R}^{-1}\left(\Sigma_{0}\right)$. According to our previous 
Fig. 4

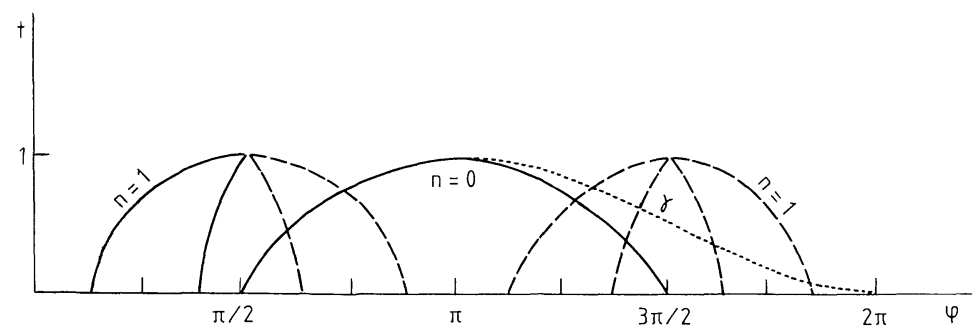

Fig. 5

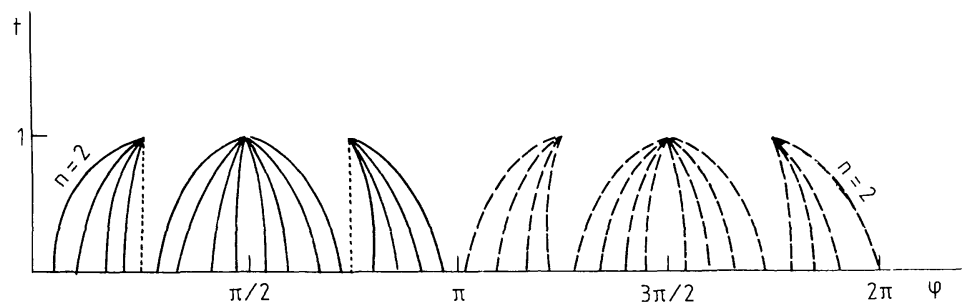

analysis the pre-image $\mathscr{R}^{-1}\left(\Sigma_{0}\right)$ in the fundamental domain $Y_{0}$ consists of "two threads" descending from the point $(\pi / 2,1)$ to the ones $(\pi / 8,0)$ and $(3 \pi / 8,0)$ (see Fig. 4). These are the pre-images of two parts of curve $\{t=-\cos \varphi\}$ going from $(\pi, 1)$ to $(\pi / 2,0)$ and $(3 \pi / 2,0)$. The full set $\Sigma_{1}$ can be obtained from these two threads by the symmetry with respect to the line $\{\varphi=\pi / 2\}$ and by a shift in $\pi$ (see broken curves in Fig. 4). As a result $\Sigma_{1}$ consists of two groups of threads, four threads in each, descending from the points $(\pi / 2,1)$ and $(3 \pi / 2,1)$.

To construct $\mathscr{R}^{-1}\left(\Sigma_{1}\right) \cap Y_{0}$ note that the curve $\gamma=\left\{t=\sin ^{2}(\varphi / 2), \pi \leqq \varphi \leqq 2 \pi\right\}$ intersects the threads in $\Sigma_{1}$ descending from the point $(3 \pi / 2,1)$. The inverse map $\mathscr{R}^{-1}$ "cut off" the upper parts of these threads lying above the curve $\gamma$ and "tie together" their lower parts lying below the curve $\gamma$ at the point $(\pi / 2,1)$. So in $\Sigma_{2}$ there are four threads descending from the point $(\pi / 2,1)$ to the points $(\varphi=9 \pi / 32$; $11 \pi / 32 ; 13 \pi / 32 ; 15 \pi / 32, t=0)$ and four symmetric threads with respect to the line $\{\varphi=\pi / 2\}$ descending from $(\pi / 2,1)$ to $(\varphi=17 \pi / 32 ; 19 \pi / 32 ; 21 \pi / 32 ; 23 \pi / 32, t=0)$. Similarly eight threads descend from the point $(3 \pi / 2,1)$ (see Fig. 5). Moreover, there are four threads in the fundamental domain $Y_{0}$ descending from the point $(\pi / 4,1)$ which are pre-images of the threads descending from $(\pi / 2,1)$ in $\Sigma_{1}$. By symmetry we have also similar "bunches of threads" descending from the points $(3 \pi / 4,1) ;(5 \pi / 4,1) ;(7 \pi / 4,1)$ (see Fig. 5).

Continuing this procedure one can see that in $\Sigma_{n}$ there are bunches of $2^{-j+1} \cdot 4^{n-1}$ threads descending from the points

$$
\left(\frac{\pi}{2^{j+1}}, 1\right) ;\left(\frac{3 \pi}{2^{j+1}}, 1\right) ; \ldots ;\left(\frac{\left(2^{j+2}-1\right) \pi}{2^{j+1}}, 1\right), \quad j=0,1, \ldots, n-1 .
$$

A characteristic feature of the distribution of zeroes of the partition function $\Xi_{n}$ is that at any fixed $T$ (i.e. fixed $t_{0}$ ) the distance between any two neighboured zeroes on the circle $\left\{\left|z_{0}\right|=1, z_{0}=\exp (-2 h / b T)\right\}$ does not exceed $2 \pi b^{-n}$, so they are dense on the circle $\left\{\left|z_{0}\right|=1\right\}$ in the limit $n \rightarrow \infty$. Note that in the Ising model on $Z^{d}$ at high 
temperatures the zeroes of the partition function lie near the point $z=-1$, $z=\exp (-2 h / T)$ and are not dense on the circle $\{|z|=1\}$.

Figures 4, 5 were obtained with the help of a computer. The algorithm of these computations is presented in Appendix $C$.

\section{Conclusion}

The Migdal-Kadanoff approximation is often applied in the consideration of various models of statistical physics and quantum field theory. It acts most effectively near the low critical dimension, when the critical temperature is low. At the same time, as is seen from the present paper, this approximation can demonstrate an anomalous behaviour of the thermodynamical function (in our case of the susceptibility) in the high temperature region as well (see also [12]). This phenomenon is strongly connected with the anomalous behaviour of the zeroes of the partition function which, in the thermodynamical limit, form an everywheredense set on the unit circle $|z|=1$.

\section{Appendix A}

In this Appendix we shall prove Proposition 1, dividing the proof into several lemmas. For convenience, further we shall use new variables $v_{n}$ and $\eta_{n}$ of the RG equations which are related to $t_{n}$ and $z_{n}$ by

$$
z_{n}=\exp \left(-\eta_{n}\right) ; \quad t_{n}=\exp \left(-v_{n}\right) .
$$

In these variables the $\mathrm{RG}$ equations (3) are rewritten in the form

$$
\begin{gathered}
\eta_{n+1}=-\ln \left[\frac{\exp \left(-b \eta_{n}\right)+\exp \left(-b v_{n}\right)}{\exp \left(b \eta_{n}\right)+\exp \left(-b v_{n}\right)}\right], \\
v_{n+1}=-\ln \left[\frac{\cosh \left(b \eta_{n}\right)+1}{\cosh \left(b \eta_{n}\right)+\cosh \left(b v_{n}\right)}\right],
\end{gathered}
$$

with the initial conditions

$$
\eta_{0}=2 h / b T ; \quad v_{0}=2 J / b T .
$$

Now we prove in detail properties of the trajectories $\left(\eta_{n}, v_{n}\right)$ which were formulated in terms of $\left(z_{n}, t_{n}\right)$ in Sect. 2.

When $h=0$ from (18) the recurrence relation follows:

$$
v_{n+1}=f\left(v_{n}\right)=-\ln \left[2\left(1+\cosh \left(b v_{n}\right)\right)^{-1}\right]
$$

[see (5)], for which the following statements hold:

Lemma 1. Let $\left\{v_{n}>0, n=0,1, \ldots\right\}$ be a sequence for which (20) holds. Then there exists a unique fixed point $v_{c}>0$ such that $v_{c}=f\left(v_{c}\right), f^{\prime}\left(v_{c}\right)>1$ and

1) if $v_{0}<v_{c}$, then $v_{0}>v_{1}>v_{2}>\ldots$ and $\lim _{n \rightarrow \infty} v_{n}=0$. Moreover there exist constants $C_{1}=C_{1}\left(v_{0}, b\right)>0$ and $C_{2}=C_{2}\left(v_{0}, b\right)>0$ such that $0<v_{n}<C_{1} \exp \left(-C_{2} 2^{n}\right)$,

2) if $v_{0}>v_{c}$, then $v_{0}<v_{1}<v_{2}<\ldots$ and $\lim _{n \rightarrow \infty} v_{n}=\infty$. 
Proof. Using elementary properties of the hyperbolic trigonometric function, the following relations and estimates can be directly obtained:

$$
\begin{gathered}
f(v)=2 \ln \cosh (b v / 2) ; \\
f^{\prime}(v)=b \tanh (b v / 2)>0 ; \quad f^{\prime \prime}(v)=\left(b^{2} / 2\right) \cosh ^{-2}(b v / 2)>0 ; \\
f(0)=f^{\prime}(0)=0 ; \quad f^{\prime \prime}(0)=b^{2} / 2 ; \quad \lim _{v \rightarrow \infty}(f(v) / v)=b>1 .
\end{gathered}
$$

Consequently $f(v) \sim b^{2} v^{2} / 4<v$, when $v \ll 1$ and $f(v) \sim b v>v$, when $v>1$, which means that function $f(v)-v$ changes its sign and there exists $v_{c}>0$ such that $f\left(v_{c}\right)$ $-v_{c}=0$. Since this function is strictly convex there can be only two simple zeroes of it: $v=0$ and $v=v_{c}$, so $v_{c}$ is the only critical point and $f^{\prime}\left(v_{c}\right)>1$. Moreover $f(v)<v$, when $0<v<v_{c}$, so $v_{1}=f\left(v_{0}\right)<v_{0}, v_{2}=f\left(v_{1}\right)<v_{1}$, etc., when $0<v_{0}<v_{c}$ and, similarly, $v_{0}<v_{1}<v_{2}<\ldots$, when $v_{0}>v_{c}$.

Let us estimate now the rate of the convergence of $v_{n}$, when $v_{0}<v_{c}$. First, note that as $v_{0}>v_{1}>\ldots>0, \lim _{n \rightarrow \infty} v_{n}=v_{*}$ exists and $f\left(v_{*}\right)=\lim _{n \rightarrow \infty} f\left(v_{n}\right)=\lim _{n \rightarrow \infty} v_{n}=v_{*}$, so $v_{*}=0$. Second, since $f^{\prime}(0)=f(0)=0, C=C\left(v_{0}, b\right)$ exist such that $f(v) \leqq C v^{2}$ for $0<v$ $<v_{0}$. So $v_{n+1} \leqq \mathrm{C} v_{n}^{2}, \mathrm{C} v_{n+1} \leqq\left(\mathrm{C} v_{n}\right)^{2}$, and consequently

$$
C v_{n} \leqq\left(C v_{n-1}\right)^{2} \leqq\left(C v_{n-2}\right)^{4} \leqq \ldots \leqq\left(C v_{m}\right)^{2^{n-m}}
$$

Choose $m$ such that $C v_{m}<1$. Then $C v_{m}=\exp \left(-C_{1}\right), C_{1}>0$ and $C v_{n} \leqq\left(C v_{m}\right)^{2^{n-m}}$ $=\exp \left(-C_{1} 2^{n-m}\right)=\exp \left(-C_{2} 2^{n}\right), C_{2}=C_{1} 2^{-m}$. Thus $v_{n} \leqq C^{-1} \exp \left(-C_{2} 2^{n}\right)$, as was stated. Lemma 1 is proved.

In Lemmas $2-6\left\{\left(\eta_{n}, v_{n}\right), n=0,1, \ldots\right\}$ will be a sequence which satisfies recurrent formulae (18), (19).

Lemma 2. 1) If $\eta_{n}, v_{n} \geqq 0$, then $b \eta_{n} \leqq \eta_{n+1} \leqq 2 b \eta_{n}, v_{n+1} \leqq f\left(v_{n}\right)$,

2) if $0<v_{0}<v_{c}$, then $v_{0}>v_{1}>\ldots$, and there exist constants $C_{1}=C_{1}\left(v_{0}, b\right)>0$ and $C_{2}=C_{2}\left(v_{0}, b\right)>0$ such that

$$
0<v_{n}<C_{1} \exp \left(-C_{2} 2^{n}\right)
$$

Proof. 1) Validity of the statement 1) follows directly from the relations

$$
\begin{gathered}
\min _{v_{n}>0} \exp \left(-\eta_{n+1}\right)=\min _{v_{n}>0} \frac{\exp \left(-b \eta_{n}\right)+\exp \left(-b v_{n}\right)}{\exp \left(b \eta_{n}\right)+\exp \left(-b v_{n}\right)}=\exp \left(-2 b \eta_{n}\right) \\
\max _{v_{n}>0} \exp \left(-\eta_{n+1}\right)=\exp \left(-b \eta_{n}\right) \\
\min _{\eta_{n}>0} \exp \left(-v_{n+1}\right)=\min _{\eta_{n}>0} \frac{\cosh \left(b \eta_{n}\right)+1}{\cosh \left(b \eta_{n}\right)+\cosh \left(b v_{n}\right)}=\frac{2}{1+\cosh \left(b v_{n}\right)}
\end{gathered}
$$

2) Inequality $v_{n+1} \leqq f\left(v_{n}\right)$ and Lemma 1 imply $v_{c}>v_{0}>v_{1}>\ldots$. To obtain (21) we introduce an auxiliary sequence $\left\{\bar{v}_{n}, n=0,1, \ldots\right\}$, which is defined by the recurrent equation $\bar{v}_{n+1}=f\left(\bar{v}_{n}\right), \bar{v}_{0}=v_{0}$. Let us prove by induction that $v_{n} \leqq \bar{v}_{n}$. When $n=0$ this fact follows from the definition of $v_{0}$. Let it be valid for some number $n$. Due to statement 1) of the present lemma $v_{n+1} \leqq f\left(v_{n}\right)$, and since function $f(v)$ is monotonous, we have

$$
f\left(v_{n}\right) \leqq f\left(\bar{v}_{n}\right) \leqq \bar{v}_{n+1}
$$


Consequently, $v_{n+1} \leqq \bar{v}_{n+1}$, which completes the inductive proof. Now according to Lemma 1 for $v_{0}<v_{c}$ we have that $\bar{v}_{n} \leqq C_{1} \exp \left(-C_{2} 2^{n}\right)$, and hence $v_{n} \leqq C_{1} \exp \left(-C_{2} 2^{n}\right)$ which ends proof of Lemma 2 .

Now we rewrite the formula for the susceptibility in new variables. According to (10) we have

$$
\chi=-\frac{\partial^{2} F}{\partial h^{2}}=\frac{T}{2} \sum_{n=0}^{\infty}(2 b)^{-n} \frac{\partial^{2}}{\partial h^{2}}\left[\ln \left(1+z_{n}^{b} t_{n}^{b}\right)\right],
$$

or in the new variables

$$
\chi=\frac{T}{2} \sum_{n=0}^{\infty}(2 b)^{-n} \frac{\partial^{2}}{\partial h^{2}}\left[\ln \left(1+\exp \left(-b \eta_{n}-b v_{n}\right)\right)\right] .
$$

By differentiating twice in $h$ we obtain the following formula:

$$
\begin{aligned}
\chi= & \frac{T}{2} \sum_{n=0}^{\infty}(2 b)^{-n}\left\{\frac{b^{2}}{2}\left(1+\cosh \left(b \eta_{n}+b v_{n}\right)\right)^{-1}\left(\frac{\partial \eta_{n}}{\partial h}+\frac{\partial v_{n}}{\partial h}\right)^{2}\right. \\
& \left.-b\left(1+\exp \left(b \eta_{n}+b v_{n}\right)\right)^{-1}\left(\frac{\partial^{2} \eta_{n}}{\partial h^{2}}+\frac{\partial^{2} v_{n}}{\partial h^{2}}\right)\right\} .
\end{aligned}
$$

The following four lemmas contain various estimates concerning the derivatives in formula (22).

Lemma 3. (estimations of the first derivatives of the $\mathrm{RG}$ transformations). If $\eta_{n}$, $v_{n} \geqq 0$, then

$$
\begin{aligned}
& \text { 1. }\left|\frac{\partial \eta_{n+1}}{\partial \eta_{n}}\right| \leqq 2 b ; \\
& \text { 2. }\left|\frac{\partial \eta_{n+1}}{\partial v_{n}}\right| \leqq b \tanh \left(b \eta_{n}\right) ; \\
& \text { 3. }\left|\frac{\partial v_{n+1}}{\partial \eta_{n}}\right| \leqq b^{2} \tanh ^{2}\left(b v_{n}\right) \tanh \left(b \eta_{n}\right) ; \\
& \text { 4. }\left|\frac{\partial v_{n+1}}{\partial v_{n}}\right| \leqq b \tanh \left(b v_{n}\right) ; \\
& \text { 5. }\left|\frac{\partial \eta_{n+1}}{\partial \eta_{n}}-\frac{2 b}{1+\exp \left(-b v_{n}\right)}\right| \leqq b \tanh \left(b v_{n} / 2\right) \tanh ^{2}\left(b \eta_{n}\right) .
\end{aligned}
$$

Proof. The estimates 1-4 can be obtained by differentiating in $\eta_{n}$ and $v_{n}$ of both Eq. (18) and using properties of the hyperbolic trigonometric functions.

For statement 1 we have

$$
\left|\frac{\partial \eta_{n+1}}{\partial \eta_{n}}\right|=b\left|\frac{\exp \left(b v_{n}\right)+\cosh \left(b \eta_{n}\right)}{\cosh \left(b v_{n}\right)+\cosh \left(b \eta_{n}\right)}\right| \leqq 2 b,
$$

because $\exp \left(b v_{n}\right)=\cosh \left(b v_{n}\right)+\sinh \left(b v_{n}\right)$. Estimates 2-4 can be obtained similarly.

Let's prove 5. Using (23) we have

$$
\frac{\partial \eta_{n+1}}{\partial \eta_{n}}-\frac{2 b}{1+\exp \left(-b v_{n}\right)}=\frac{b \tanh \left(b v_{n} / 2\right)\left[1-\cosh \left(b \eta_{n}\right)\right]}{\cosh \left(b v_{n}\right)+\cosh \left(b \eta_{n}\right)},
$$


and the required estimate is a consequence of the inequality:

$$
\left|1-\cosh \left(b \eta_{n}\right)\right|=\sinh ^{2}\left(b \eta_{n}\right)\left[1+\cosh \left(b \eta_{n}\right)\right]^{-1} \leqq \tanh ^{2}\left(b \eta_{n}\right) \cosh \left(b \eta_{n}\right) .
$$

Lemma 4. (estimations of the second derivatives of the RG transformations). If $\eta_{n}$, $v_{n} \geqq 0$, then
1. $\left|\frac{\partial^{2} \eta_{n+1}}{\partial \eta_{n}^{2}}\right| \leqq b^{2} \tanh \left(b v_{n}\right) \tanh \left(b \eta_{n}\right) ;$
2. $\left|\frac{\partial^{2} \eta_{n+1}}{\partial \eta_{n} \partial v_{n}}\right| \leqq b^{2}$
3. $\left|\frac{\partial^{2} \eta_{n+1}}{\partial v_{n}^{2}}\right| \leqq b^{2} \tanh \left(b v_{n}\right) \tanh \left(b \eta_{n}\right)$;
4. $\left|\frac{\partial^{2} v_{n+1}}{\partial \eta_{n}^{2}}\right| \leqq b^{2} \tanh ^{2}\left(b v_{n}\right)$;
5. $\left|\frac{\partial^{2} v_{n+1}}{\partial \eta_{n} \partial v_{n}}\right| \leqq b^{2} \tanh \left(b v_{n}\right) \tanh \left(b \eta_{n}\right)$
6. $\left|\frac{\partial^{2} v_{n+1}}{\partial v_{n}^{2}}\right| \leqq b^{2}$.

Proof. These estimates are obtained in the same way as in the previous lemma. For example, differentiating (23) in $\eta_{n}$, we have

$$
\frac{\partial^{2} \eta_{n+1}}{\partial \eta_{n}^{2}}=-b^{2} \frac{\sinh \left(b v_{n}\right) \sinh \left(b \eta_{n}\right)}{\left[\cosh \left(b v_{n}\right)+\cosh \left(b \eta_{n}\right)\right]^{2}},
$$

so

$$
\left|\frac{\partial^{2} \eta_{n+1}}{\partial \eta_{n}^{2}}\right| \leqq b^{2} \frac{\sinh \left(b v_{n}\right) \sinh \left(b \eta_{n}\right)}{2 \cosh \left(b v_{n}\right) \cosh \left(b \eta_{n}\right)}<b^{2} \tanh \left(b v_{n}\right) \tanh \left(b \eta_{n}\right)
$$

The remaining five estimates can be obtained analogously.

For convenience, further we shall use the notation

$$
A_{n}=\prod_{j=0}^{n-1} \frac{2}{1+\exp \left(-b v_{j}\right)} .
$$

Lemma 5. (estimations of the first derivatives in $h$ ). If $\eta_{0} \geqq 0$ and $0<v_{0}<v_{c}$, then

1. $\left|\frac{\partial v_{n}}{\partial h}\right| \leqq \frac{2}{b T}(4 b)^{n} \tanh \left(b v_{n-1}\right) \tanh \left(b \eta_{n-1}\right)$,

where $v_{-1}=v_{0}, \eta_{-1}=\eta_{0}$ by definition,

2. there exist constants $C_{0}=C_{0}\left(v_{0}, b\right)>0$ and $C=C\left(v_{0}, b\right)>0$ such that

$$
\left|\frac{\partial v_{n}}{\partial h}\right| \leqq C_{0}|h| \exp \left(-C 2^{n}\right)
$$

3. $\left|\frac{\partial \eta_{n}}{\partial h}\right| \leqq \frac{2}{b T}(4 b)^{n}$ 

4. $\left|\frac{\partial \eta_{n}}{\partial h}-\frac{2}{b T} A_{b} b^{b}\right| \leqq \frac{2}{b T} A_{n} b^{n} \sum_{j=0}^{n-1} 4^{j+1} \tanh \left(b v_{j-1}\right) \tanh ^{2}\left(b \eta_{j}\right)$
5. $\left|\frac{\partial \eta_{n}}{\partial h} \cdot \frac{b T}{2 A_{n} b^{n}}-1\right| \leqq C_{0} h^{2}$.

Proof. We shall prove these statements by induction. It is obvious that all inequalities are valid when $n=0$, which gives us an initial step of the induction. Let's assume their validity for some $n>0$ and show it for $n+1$.

We have:

$$
\begin{aligned}
& \frac{\partial v_{n+1}}{\partial h}=\frac{\partial v_{n+1}}{\partial v_{n}} \cdot \frac{\partial v_{n}}{\partial h}+\frac{\partial v_{n+1}}{\partial \eta_{n}} \cdot \frac{\partial \eta_{n}}{\partial h} \\
& \frac{\partial \eta_{n+1}}{\partial h}=\frac{\partial \eta_{n+1}}{\partial v_{n}} \cdot \frac{\partial v_{n}}{\partial h}+\frac{\partial \eta_{n+1}}{\partial \eta_{n}} \cdot \frac{\partial \eta_{n}}{\partial h} .
\end{aligned}
$$

Using these formulae we prove 1-5.

1. Applying the estimates of Lemma 3 and our inductive assumptions to (24), we obtain

$$
\begin{aligned}
&\left|\frac{\partial v_{n+1}}{\partial h}\right| \leqq b \tanh \left(b v_{n}\right) \frac{2}{b T}(4 b)^{n} \tanh \left(b v_{n-1}\right) \tanh \left(b \eta_{n-1}\right) \\
&+\frac{2}{b T}(4 b)^{n} b \tanh ^{2}\left(b v_{n}\right) \tanh \left(b \eta_{n}\right) .
\end{aligned}
$$

According to Lemma $2 \eta_{n} \geqq \eta_{n-1}$, and so $\tanh \left(b \eta_{n}\right) \geqq \tanh \left(b \eta_{n-1}\right)$. Moreover, $0<\tanh \left(b v_{n-1}\right)<1$, and consequently

$$
\left|\frac{\partial v_{n+1}}{\partial h}\right| \leqq \frac{2}{b T}(4 b)^{n+1} \tanh \left(b v_{n}\right) \tanh \left(b \eta_{n}\right) . \quad \text { Q.E.D. }
$$

2. Continuing the last inequality we have

$$
\left|\frac{\partial v_{n+1}}{\partial h}\right| \leqq \frac{2 b}{T}(4 b)^{n+1} v_{n} \eta_{n}
$$

which enables us to prove estimate 2. Namely, according to Lemma 2, the sequence $v_{n}$ decreases with the superexponential rate as $n \rightarrow \infty$, while $\eta_{n}$ increases as a simple exponent; hence $\left|\partial v_{n} / \partial h\right|$ decreases superexponentially as well. So

$$
0<v_{n} \leqq C_{0} \exp \left(-C 2^{n}\right) ; \quad \eta_{n} \leqq 2 h(2 b)^{n} / b T,
$$

and consequently

$$
\left|\frac{\partial v_{n+1}}{\partial h}\right| \leqq(4 b)^{n+1}(2 b)^{n}(2 / T)^{2}|h| C_{0} \exp \left(-C 2^{n+1} / 2\right) .
$$

Let $C_{1}=C / 4$ and $C_{2}=\max (8 b)^{2 n}(2 / T)^{2} C_{0} \exp \left(-C 2^{n+1} / 4\right)$, then

$$
\left|\frac{\partial v_{n+1}}{\partial h}\right| \leqq C_{2}|h| \exp \left(-C_{1} 2^{n+1}\right) \text { Q.E.D. }
$$


3. Analogously, applying Lemma 3 to (25) we obtain an estimate for $\left|\partial \eta_{n+1} / \partial h\right|$.

4. Furthermore,

$$
\begin{aligned}
\mid \frac{\partial \eta_{n+1}}{\partial h} & -\frac{2 b}{1+\exp \left(-b v_{n}\right)} \cdot \frac{\partial \eta_{n}}{\partial h} \mid \\
& \leqq\left|\frac{\partial \eta_{n+1}}{\partial \eta_{n}}-\frac{2 b}{1+\exp \left(-b v_{n}\right)}\right| \cdot\left|\frac{\partial \eta_{n}}{\partial h}\right|+\left|\frac{\partial \eta_{n+1}}{\partial v_{n}} \cdot \frac{\partial v_{n}}{\partial h}\right| \\
& \leqq \frac{2}{b T}(4 b)^{n+1} \tanh \left(b v_{n-1}\right) \tanh ^{2}\left(b \eta_{n}\right)
\end{aligned}
$$

To obtain the last inequality we used the fact that $v_{n-1} \geqq v_{n}$ and, hence $\tanh \left(b v_{n}\right)$ $\leqq \tanh \left(b v_{n-1}\right)$. Now under the inductive assumption and previous estimation we have

$$
\begin{aligned}
\left|\frac{\partial \eta_{n+1}}{\partial h}-\frac{2}{b T} A_{n+1} b^{n+1}\right| \leqq & \frac{2 b}{1+\exp \left(-b v_{n}\right)}\left|\frac{\partial \eta_{n}}{\partial h}-\frac{2}{b T} A_{n} b^{n}\right| \\
& +\frac{2}{b T}(4 b)^{n+1} \tanh \left(b v_{n-1}\right) \tanh ^{2}\left(b \eta_{n-1}\right) \\
\leqq & \frac{2}{b T} A_{n+1} b^{n+1} \sum_{j=0}^{n-1} 4^{j+1} \tanh \left(b v_{j-1}\right) \tanh ^{2}\left(b \eta_{j}\right) \\
& +\frac{2}{b T}(4 b)^{n+1} \tanh \left(b v_{n-1}\right) \tanh ^{2}\left(b \eta_{n}\right) \\
\leqq & \frac{2}{b T} A_{n+1} b^{n+1} \sum_{j=0}^{n} 4^{j+1} \tanh \left(b v_{j-1}\right) \tanh ^{2}\left(b \eta_{j}\right)
\end{aligned}
$$

where the last inequality is valid because $A_{n+1}>1$. Q.E.D.

5. To prove the last estimate we note that by (26)

$$
\begin{aligned}
& \sum_{j=0}^{n-1} 4^{j+1} \tanh \left(b v_{j-1}\right) \tanh ^{2}\left(b \eta_{j}\right) \leqq \sum_{j=0}^{n-1} 4^{j+1} b^{3} v_{j-1} \eta_{j}^{2} \\
& \leqq \sum_{j=0}^{\infty} 4^{j+1} b^{3} C_{0} \exp \left(-C 2^{j+1}\right)(2 b)^{2 j}(2 h / b T)^{2}=C_{1} h^{2}
\end{aligned}
$$

Hence the required result follows from 4 . So Lemma 5 is proved.

Lemma 6 (estimations of the second derivatives in $h$ ). If $\eta_{0} \geqq 0$ and $0<v_{0}<v_{c}$, then

1. $\left|\frac{\partial^{2} v_{n}}{\partial h^{2}}\right| \leqq \frac{4}{(b T)^{2}}(4 b)^{2 n} \tanh \left(b v_{n-2}\right)$,

where $v_{-2}=v_{-1}=v_{0}$ by definition, 
2. there exist constants $C_{0}=C_{0}\left(v_{0}, b\right)>0, C=C\left(v_{0}, b\right)>0$, such that $\left|\partial^{2} v_{n} / \partial h^{2}\right|$ $\leqq C_{0} \exp \left(-C 2^{n}\right)$
3. $\left|\frac{\partial^{2} \eta_{n}}{\partial h^{2}}\right| \leqq\left(\frac{2}{b T}\right)^{2}(4 b)^{2 n}$,
4. $\left|\frac{\partial^{2} \eta_{n}}{\partial h^{2}}\right| \leqq\left(\frac{2}{b T}\right)^{2} A_{n} b^{n} \sum_{j=0}^{n-1}(4 b)^{2 j+2} \tanh \left(b v_{j-2}\right) \tanh \left(b \eta_{j}\right)$,
5. $\left|\frac{\partial^{2} \eta_{n}}{\partial h^{2}}\right| \leqq C_{0} A_{n} b^{n}|h|$.

Proof. As in the previous lemma, all the statements are valid for $n=0$, because $\partial^{2} v_{0} / \partial h^{2}=\partial^{2} \eta_{0} / \partial h^{2}=0$. Let's assume validity of these statements for some $n>0$ and prove the same for $n+1$.

Differentiating formulae (24), (25) in $h$ we obtain

$$
\begin{aligned}
\frac{\partial^{2} v_{n+1}}{\partial h^{2}}= & \frac{\partial v_{n+1}}{\partial v_{n}} \cdot \frac{\partial^{2} v_{n}}{\partial h^{2}}+\frac{\partial v_{n+1}}{\partial \eta_{n}} \cdot \frac{\partial^{2} \eta_{n}}{\partial h^{2}}+\frac{\partial^{2} v_{n+1}}{\partial v_{n}^{2}} \cdot\left(\frac{\partial v_{n}}{\partial h}\right)^{2} \\
& +2 \frac{\partial^{2} v_{n+1}}{\partial v_{n} \partial \eta_{n}} \cdot \frac{\partial v_{n}}{\partial h} \cdot \frac{\partial \eta_{n}}{\partial h}+\frac{\partial^{2} v_{n+1}}{\partial \eta_{n}^{2}} \cdot\left(\frac{\partial \eta_{n}}{\partial h}\right)^{2}, \\
\frac{\partial^{2} \eta_{n+1}}{\partial h}= & \frac{\partial \eta_{n+1}}{\partial v_{n}} \cdot \frac{\partial^{2} v_{n}}{\partial h^{2}}+\frac{\partial \eta_{n+1}}{\partial \eta_{n}} \cdot \frac{\partial^{2} \eta_{n}}{\partial h^{2}}+\frac{\partial^{2} \eta_{n+1}}{\partial v_{n}^{2}} \cdot\left(\frac{\partial v_{n}}{\partial h}\right)^{2} \\
& +2 \frac{\partial^{2} \eta_{n+1}}{\partial v_{n} \partial \eta_{n}} \cdot \frac{\partial v_{n}}{\partial h} \cdot \frac{\partial \eta_{n}}{\partial h}+\frac{\partial^{2} \eta_{n+1}}{\partial \eta_{n}^{2}} \cdot\left(\frac{\partial \eta_{n}}{\partial h}\right)^{2} .
\end{aligned}
$$

1. Now using the estimates obtained in Lemmas 3-5 and our inductive assumptions by direct calculations we have the following inequalities:

$$
\begin{aligned}
\left|\frac{\partial^{2} v_{n+1}}{\partial h^{2}}\right| \leqq & (2 / b T)^{2} b(4 b)^{2 n} \tanh \left(b v_{n}\right) \tanh \left(b v_{n-2}\right) \\
& +(2 / b T)^{2} b(4 b)^{2 n} \tanh \left(b \eta_{n}\right) \tanh ^{2}\left(b v_{n}\right) \\
& +(2 / b T)^{2} b^{2}(4 b)^{2 n} \tanh ^{2}\left(b v_{n-1}\right) \tanh ^{2}\left(b \eta_{n-1}\right) \\
& +(2 / b T)^{2} 2 b^{2}(4 b)^{2 n} \tanh \left(b v_{n}\right) \tanh \left(b v_{n-1}\right) \tanh \left(b \eta_{n}\right) \tanh \left(b \eta_{n-1}\right) \\
& +(2 / b T)^{2} b^{2}(4 b)^{2 n} \tanh \left(b v_{n}\right) \leqq(2 / b T)^{2}(4 b)^{2 n+2} \tanh \left(b v_{n-1}\right) .
\end{aligned}
$$

(Here we used also the fact from Lemma 2 that $v_{n-1}>v_{n}>0$ and consequently $\tanh \left(b v_{n-1}\right)>\tanh \left(b v_{n}\right)>0$.) Q.E.D.

2. From (21) and the previous estimate it follows that

$$
\left|\frac{\partial^{2} v_{n}}{\partial h^{2}}\right| \leqq\left(\frac{2}{b T}\right)^{2}(4 b)^{2 n} C_{0} \exp \left(-C 2^{n-2}\right) \leqq C_{1} \exp \left(-C_{2} 2^{n}\right) \text {. Q.E.D. }
$$


3. As in the first estimation of this lemma one can easily obtain the inequalities:

$$
\begin{aligned}
& \left|\frac{\partial^{2} \eta_{n+1}}{\partial h^{2}}\right| \leqq(2 / b T)^{2} b(4 b)^{2 n} \tanh \left(b \eta_{n}\right) \tanh \left(b v_{n-2}\right)+\left|\frac{\partial \eta_{n+1}}{\partial \eta_{n}} \cdot \frac{\partial^{2} \eta_{n}}{\partial h^{2}}\right| \\
& \quad+(2 / b T)^{2} b^{2}(4 b)^{2 n} \tanh \left(b v_{n}\right) \tanh ^{2}\left(b v_{n-1}\right) \tanh \left(b \eta_{n}\right) \tanh ^{2}\left(b \eta_{n-1}\right) \\
& \quad+(2 / b T)^{2} 2 b^{2}(4 b)^{2 n} \tanh \left(b v_{n-1}\right) \tanh \left(b \eta_{n-1}\right) \\
& \quad+(2 / b T)^{2} b^{2}(4 b)^{2 n} \tanh \left(b v_{n}\right) \tanh \left(b \eta_{n}\right) \leqq\left|\frac{\partial \eta_{n+1}}{\partial \eta_{n}} \cdot \frac{\partial^{2} \eta_{n}}{\partial h^{2}}\right| \\
& \quad+(2 / b T)^{2} 6 b^{2}(4 b)^{2 n} \tanh \left(b v_{n-2}\right) \tanh \left(b \eta_{n}\right) .
\end{aligned}
$$

Because $\left|\partial \eta_{n+1} / \partial \eta_{n}\right| \leqq 2 b$ (according to Lemma 3) and $\left|\partial^{2} \eta_{n} / \partial h^{2}\right| \leqq(2 / b T)^{2}(4 b)^{2 n}$ (according to inductive assumption) we have finally the required estimate

$$
\left|\frac{\partial^{2} \eta_{n+1}}{\partial h^{2}}\right| \leqq 2 b(4 b)^{2 n}\left(\frac{2}{b T}\right)^{2}+6 b^{2}(4 b)^{2 n}\left(\frac{2}{b T}\right)^{2} \leqq(4 b)^{2 n+2}\left(\frac{2}{b T}\right)^{2} \text {. Q.E.D. }
$$

4. On the other hand according to estimate 5 of Lemma 3

$$
\left|\frac{\partial \eta_{n+1}}{\partial \eta_{n}}\right| \leqq \frac{2 b}{1+\exp \left(-b v_{n}\right)}+b \tanh \left(b v_{n}\right) \tanh ^{2}\left(b \eta_{n}\right)
$$

which allows us to write the chain of inequalities continuing (27),

$$
\begin{aligned}
\left|\frac{\partial^{2} \eta_{n+1}}{\partial h^{2}}\right| \leqq & \frac{2 b}{1+\exp \left(-b v_{n}\right)} \cdot\left|\frac{\partial^{2} \eta_{n}}{\partial h^{2}}\right| \\
& +(2 / b T)^{2} b^{2}(4 b)^{2 n} \tanh \left(b v_{n}\right) \tanh ^{2}\left(b \eta_{n}\right) \\
& +(2 / b T)^{2} 6 b^{2}(4 b)^{2 n} \tanh \left(b \eta_{n}\right) \tanh \left(b v_{n-2}\right) \\
\leqq & \frac{2 b}{1+\exp \left(-b v_{n}\right)} \cdot\left|\frac{\partial^{2} \eta_{n}}{\partial h^{2}}\right| \\
& +\left(\frac{2}{b T}\right)^{2}(4 b)^{2 n+2} \tanh \left(b v_{n-2}\right) \tanh \left(b \eta_{n}\right) .
\end{aligned}
$$

Now applying inductive assumption 4 of the present lemma, we obtain

$$
\begin{aligned}
\left|\frac{\partial^{2} \eta_{n+1}}{\partial h^{2}}\right| \leqq & (2 / b T)^{2} A_{n+1} b^{n+1} \sum_{j=0}^{n-1}(4 b)^{2 j+2} \tanh \left(b v_{j-2}\right) \tanh \left(b \eta_{j}\right) \\
& +(2 / b T)^{2}(4 b)^{2 n+2} \tanh \left(b v_{n-2}\right) \tanh \left(b \eta_{n}\right) \\
\leqq & (2 / b T)^{2} A_{n+1} b^{n+1} \sum_{j=0}^{n}(4 b)^{2 j+2} \tanh \left(b v_{j-2}\right) \tanh \left(b \eta_{j}\right) . \quad \text { Q.E.D. }
\end{aligned}
$$

5. The last statement follows directly from 4 and (26) (cf. the proof of the statement 5 of Lemma 5). Lemma 6 is proved.

To prove Proposition 1 we shall need some estimates of the dependence of the $\mathrm{RG}$ trajectories on $h$ and $n$. Let's unite them into the following 
Lemma 7. Let $0<v_{0}<v_{c}$ be fixed and the sequence $\left\{\left(v_{n}(h), \eta_{n}(h), n=0,1, \ldots\right\}\right.$ be defined by recurrent relations (18), (19). Then there exist constants $C_{0}=C_{0}\left(v_{0}, b\right)>0$, $C=C\left(v_{0}, b\right)>0$ such that

1. $\left|v_{n}(h)-v_{n}(0)\right| \leqq C_{0} \exp \left(-C 2^{n}\right) h^{2}$.

2. $\lim _{n \rightarrow \infty} \prod_{j=0}^{n-1} 2\left[1+\exp \left(-b v_{j}(h)\right)\right]^{-1}=\lim _{n \rightarrow \infty} A_{n}(h)=A(h)>0$ exists and $|A(h)-A|$ $\leqq C_{0} h^{2}$, where $A=A(0)$,

$$
\left|A_{n}(h)-A(h)\right| \leqq C_{0} \exp \left(-C 2^{n}\right) .
$$

3. $\lim _{n \rightarrow \infty} b^{-n}\left(\partial \eta_{n}(h) / \partial h\right)=B(h)$ exists and

$$
\begin{gathered}
\left|b^{-n}\left(\partial \eta_{n}(h) / \partial h\right)-B(h)\right| \leqq C_{0} \exp \left(-C 2^{n}\right), \\
|B(h)-2 A / b T| \leqq C_{0} h^{2} .
\end{gathered}
$$

4. $\lim _{n \rightarrow \infty} b^{-n} \eta_{n}(h)=\Psi(h)$ exists and

$$
\begin{gathered}
\left|b^{-n} \eta_{n}(h)-\Psi(h)\right| \leqq C_{0} \exp \left(-C 2^{n}\right), \\
|\Psi(h)-2 A / b T| \leqq C_{0}|h|^{3} .
\end{gathered}
$$

Note. For brevity we do not indicate the dependence of all functions on $T$.

Proof. 1) According to the Lagrange formula $v_{n}(h)-v_{n}(0)=h \frac{\partial v_{n}(\bar{h})}{\partial h}$, where $h \geqq \bar{h} \geqq 0$. By Lemma 5 ,

$$
\left|\frac{\partial v_{n}(h)}{\partial h}\right| \leqq C_{0}|\bar{h}| \exp \left(-C 2^{n}\right) \leqq C_{0}|h| \exp \left(-C 2^{n}\right),
$$

which implies

$$
\left|v_{n}(h)-v_{n}(0)\right| \leqq C_{0} h^{2} \exp \left(-C 2^{n}\right) \text {. Q.E.D. }
$$

2. Denote $a(h)=\lim _{n \rightarrow \infty} a_{n}(h)$, where

$$
a_{n}(h)=\sum_{j=0}^{n-1} \ln \left\{2\left[1+\exp \left(-b v_{j}(h)\right]^{-1}\right\} .\right.
$$

As

$$
2\left[1+\exp \left(-b v_{j}\right)\right]^{-1}=1+\tanh \left(b v_{j} / 2\right)
$$

and $0 \leqq \ln \left[1+\tanh \left(b v_{j} / 2\right)\right] \leqq b v_{j} / 2$, we obtain the following estimate:

$$
0 \leqq a(h)-a_{n}(h) \leqq \sum_{j=n}^{\infty} b v_{j} / 2 \leqq C_{0}(b / 2) \sum_{j=n}^{\infty} \exp \left(-C 2^{j}\right) \leqq C_{1} \exp \left(-C 2^{n}\right),
$$

where we have used inequality (21). Moreover

$$
\begin{aligned}
|a(h)-a(0)| & =\left|\sum_{j=0}^{\infty}\left\{\ln \left[1+\tanh \left(b v_{j}(h) / 2\right)\right]-\ln \left[1+\tanh \left(b v_{j}(0) / 2\right)\right]\right\}\right| \\
& \leqq \sum_{j=0}^{\infty}\left|v_{j}(h)-v_{j}(0)\right| \frac{b}{2} \leqq C h^{2} .
\end{aligned}
$$


Now

$$
\left|A_{n}(h)-A(h)\right|=\left|\exp \left(a_{n}(h)\right)-\exp (a(h))\right| \leqq C_{1}\left|a_{n}(h)-a(h)\right| \leqq C_{0} \exp \left(-C 2^{n}\right) .
$$

Analogously

$$
|A(h)-A| \leqq C|a(h)-a(0)| \leqq C_{0} h^{2} . \quad \text { Q.E.D. }
$$

3. Let's consider the difference of the derivatives

$$
\begin{aligned}
\Delta \eta_{n} & =\left|b^{-(n+1)} \frac{\partial \eta_{n+1}}{\partial h}-b^{-n} \frac{\partial \eta_{n}}{\partial h}\right| \\
& =\left|b^{-n}\left(b^{-1} \frac{\partial \eta_{n+1}}{\partial \eta_{n}}-1\right) \frac{\partial \eta_{n}}{\partial h}+b^{-(n+1)} \frac{\partial \eta_{n+1}}{\partial v_{n}} \cdot \frac{\partial v_{n}}{\partial h}\right| .
\end{aligned}
$$

According to Lemma 3,

$$
\begin{aligned}
\left|b^{-1} \frac{\partial \eta_{n+1}}{\partial \eta_{n}}-1\right| & \leqq\left|1-2\left[1+\exp \left(-b v_{n}\right)\right]^{-1}\right|+\tanh \left(b v_{n} / 2\right) \tanh ^{2}\left(b \eta_{n}\right) \\
& \leqq \tanh \left(b v_{n} / 2\right)+\tanh \left(b v_{n} / 2\right) \leqq b v_{n} ; \\
\left|\frac{\partial \eta_{n+1}}{\partial v_{n}}\right| & \leqq b \tanh \left(b \eta_{n}\right) \leqq b .
\end{aligned}
$$

So, using Lemmas 2 and 5, we have

$$
\Delta \eta_{n} \leqq\left|b^{-(n-1)} v_{n} \frac{\partial \eta_{n}}{\partial h}\right|+\left|b^{-n} \frac{\partial v_{n}}{\partial h}\right| \leqq C_{0} \exp \left(-C 2^{n}\right),
$$

which means that $\lim _{n \rightarrow \infty} b^{-n}\left(\partial \eta_{n} / \partial h\right)=B(h)$ exists with the corresponding rate of the convergence.

Now according to Lemma 6

$$
\left|b^{-n} \frac{\partial \eta_{n}(h)}{\partial h}-b^{-n} \frac{\partial \eta_{n}(0)}{\partial h}\right| \leqq b^{-n} h \frac{\partial^{2} \eta_{n}(\bar{h})}{\partial h^{2}} \leqq C_{1} h^{2},
$$

which implies $|B(h)-B(0)| \leqq C_{1} h^{2}$. To end the proof of 3 its remains to note by Lemma 5 that

$$
B(0)=\left.\lim _{n \rightarrow \infty} b^{-n} \frac{\partial \eta_{n}}{\partial h}\right|_{h=0}=\lim _{n \rightarrow \infty} \frac{2 A_{n}}{b T}=\frac{2 A}{b T} . \quad \text { Q.E.D. }
$$

4. Using the previous estimations we have

$$
\lim _{n \rightarrow \infty} b^{-n} \eta_{n}(h)=\lim _{n \rightarrow \infty} \int_{0}^{h} b^{-n} \frac{\partial \eta_{n}\left(h^{\prime}\right)}{\partial h} d h^{\prime}=\int_{0}^{h} B\left(h^{\prime}\right) d h^{\prime}=\Psi(h),
$$

and

$$
\left|b^{-n} \eta_{n}(h)-\Psi(h)\right| \leqq C_{0}|h| \exp \left(-C 2^{n}\right)
$$

Moreover, by 3

$$
|\Psi(h)-2 A| h|/ b T|=\left|\int_{0}^{h}\left[B\left(h^{\prime}\right)-2 A / b T\right] d h\right| \leqq C_{0}|h|^{3} .
$$


Lemma 7 is proved.

Now we turn to the proof of Proposition 1. Estimates of Lemmas 5 and 6 show that $\partial v_{n} / \partial h$ and $\partial^{2} v_{n} / \partial h^{2}$ decrease with the rate $C_{0} \exp \left(-C_{1} 2^{n}\right)$, when $n \rightarrow \infty$, where $C_{0}, C_{1}$ do not depend on $h$, while $\partial \eta_{n} / \partial h$ and $\partial^{2} \eta_{n} / \partial h^{2}$ increase not faster than $C_{0}(4 b)^{2 n}$. Hence, the terms in formula (22) containing derivatives $\partial v_{n} / \partial h$ and $\partial^{2} v_{n} / \partial h^{2}$ bring the finite contribution and have a limit when $h \downarrow 0$. Let's turn to the analysis of the terms of (22) without these derivatives. One of them is

$$
\chi_{0}=-\frac{b T}{2} \sum_{n=0}^{\infty}(2 b)^{-n}\left[1+\exp \left(b \eta_{n}+b v_{n}\right)\right]^{-1} \frac{\partial^{2} \eta_{n}}{\partial h^{2}} .
$$

Due to Lemma 6

$$
\left|\frac{\partial^{2} \eta_{n}}{\partial h^{2}}\right| \leqq C_{0} A_{n} b^{n}|h| \leqq C_{1} b^{n}|h|
$$

so

$$
\left|\chi_{0}\right| \leqq C_{1} \frac{b T}{2} \sum_{n=0}^{\infty} 2^{-n}|h| \leqq C_{2}|h|
$$

and, consequently, $\lim _{h \downarrow p} \chi_{0}=0$.

The other term in (22), without the derivative of $v_{n}$, is

$$
\chi_{1}=\frac{T b^{2}}{4} \sum_{n=0}^{\infty}(2 b)^{-n}\left[1+\cosh \left(b v_{n}+b \eta_{n}\right)\right]^{-1}\left(\frac{\partial \eta_{n}}{\partial h}\right)^{2} .
$$

To investigate the behaviour of $\chi_{1}$ when $h \rightarrow 0$, we introduce an auxiliary function

$$
\begin{aligned}
\chi_{2} & =\frac{T b^{2}}{4} \sum_{n=0}^{\infty}(2 b)^{-n}\left[1+\cosh \left(b^{n+1} \Psi(h)\right)\right]^{-1}\left(b^{n} B(h)\right)^{2} \\
& =\frac{T b^{2}}{4} B^{2}(h) \sum_{n=0}^{\infty}\left(\frac{b}{2}\right)^{n}\left[1+\cosh \left(b^{n+1} \Psi(h)\right)\right]^{-1} .
\end{aligned}
$$

Then

$$
\begin{aligned}
\left|\chi_{2}-\chi_{1}\right| \leqq & \left(T b^{2} / 4\right) \sum_{n=0}^{\infty}(2 b)^{-n} \mid\left[1+\cosh \left(b \eta_{n}+b v_{n}\right)\right]^{-1} \\
& -\left[1+\cosh \left(b^{n+1} \Psi(h)\right)\right]^{-1} \mid\left(b^{n} B(h)\right)^{2} \\
& +\left(T b^{2} / 4\right) \sum_{n=0}^{\infty}(2 b)^{-n}\left[1+\cosh \left(b \eta_{n}+b v_{n}\right)\right]^{-1} \\
& \times\left|\left(\frac{\partial \eta_{n}}{\partial h}\right)^{2}-\left(b^{n} B(h)\right)^{2}\right|
\end{aligned}
$$

Using the estimates of Lemmas 2 and 7, we have

$$
\begin{aligned}
& \left|\left[1+\cosh \left(b \eta_{n}+b v_{n}\right)\right]^{-1}-\left[1+\cosh \left(b^{n+1} \Psi(h)\right)\right]^{-1}\right| \\
& \quad \leqq b v_{n}+b\left|\eta_{n}-b^{n} \Psi(h)\right| \leqq C_{0} \exp \left(-C 2^{n}\right)
\end{aligned}
$$


and

$$
\left|\left(\frac{\partial \eta_{n}}{\partial h}\right)^{2}-\left(b^{n} B(h)\right)^{2}\right| \leqq C_{0} \exp \left(-C 2^{n}\right)
$$

where $C, C_{0}>0$ do not depend on $n$ and $h$, so quantity $\left|\chi_{2}-\chi_{1}\right|$ remains finite and has a limit when $h \downarrow 0$.

To describe the asymptotics of $\chi_{2}$ when $h \downarrow 0$, we shall need some auxiliary statements which are connected with those of Proposition 1.

Lemma 8. For any $x>0, b \geqq 2$, denote

$$
\begin{gathered}
U(x)=\sum_{n=0}^{\infty}\left(\frac{b}{2}\right)^{n}\left[1+\cosh \left(b^{n} x\right)\right]^{-1}, \\
\sum_{n=-\infty}^{-1}\left\{\left[1+\cosh \left(2^{n} x\right)\right]^{-1}-\frac{1}{2}\right\} \\
+\sum_{n=0}^{\infty}\left[1+\cosh \left(2^{n} x\right)\right]^{-1}-\frac{1}{2} \log _{2} \frac{1}{x}, \text { if } b=2, \\
x^{\gamma} \sum_{n=-\infty}^{\infty}(b / 2)^{n}\left[1+\cosh \left(b^{n} x\right)\right]^{-1}, \text { if } b>2,
\end{gathered}
$$

where $\gamma=1-\log _{2}^{-1} b$. Then the function $K(x), x>0$ has the following properties: a) $K(b x)=K(x)$, b) $K(x)$ is analytic in $x>0$, c) $K(x)>0$, if $b>2$. Moreover,

$$
\begin{gathered}
\lim _{x \downarrow 0}\left[U(x)-\left(\log _{2}(1 / x)\right) / 2-K(x)\right]=0, \quad \text { if } \quad b=2, \\
\lim _{x \downarrow 0}\left[U(x)-x^{-\gamma} K(x)\right]=C_{0}, \quad\left|C_{0}\right|<\infty, \text { if } \quad b>2 .
\end{gathered}
$$

Proof. 1. Case $b=2$. Let's denote

$$
V(x)=\sum_{n=\infty}^{-1}\left\{\left[1+\cosh \left(2^{n} x\right)\right]^{-1}-\frac{1}{2}\right\}+\sum_{n=0}^{\infty}\left[1+\cosh \left(2^{n} x\right)\right]^{-1} .
$$

Since

$$
\left|\left[1+\cosh \left(2^{n} x\right)\right]^{-1}-\frac{1}{2}\right|=\frac{1}{2} \tanh ^{2}\left(2^{n-1} x\right) \leqq 2^{n} x^{2}
$$

so

$$
|V(x)-U(x)|=\left|\sum_{n=-\infty}^{-1}\left\{\left[1+\cosh \left(2^{n} x\right)\right]^{-1}-\frac{1}{2}\right\}\right| \leqq x^{2},
$$

and consequently

$$
\lim _{x \downarrow 0}|V(x)-U(x)|=0
$$

On the other hand

$$
\begin{aligned}
V(2 x) & =\sum_{n=-\infty}^{-1}\left\{\left[1+\cosh \left(2^{n+1} x\right)\right]^{-1}-\frac{1}{2}\right\}+\sum_{n=0}^{\infty}\left[1+\cosh \left(2^{n+1} x\right)\right]^{-1} \\
& =\sum_{k=-\infty}^{-1}\left\{\left[1+\cosh \left(2^{k} x\right)\right]^{-1}-\frac{1}{2}\right\}+\sum_{k=0}^{\infty}\left[1+\cosh \left(2^{k} x\right)\right]^{-1}-\frac{1}{2}
\end{aligned}
$$


which yields $V(2 x)=V(x)-\frac{1}{2}$. By definition

$$
K(x)=V(x)-\frac{1}{2} \log _{2}(1 / x) .
$$

Then

$$
K(2 x)=V(2 x)-\frac{1}{2} \log _{2}(1 / 2 x)=V(x)-\frac{1}{2}-\frac{1}{2} \log _{2}(1 / x)+\frac{1}{2}=K(x) .
$$

Since the series defining the function $V(x)$ converges in a complex neighbourhood of any point $x>0$, so this function is analytical in $x>0$ and consequently the function $K(x)$ defined by (29) is analytic too.

2. Case $b>2$. Now by $V(x)$ denote the series

$$
V(x)=\sum_{n=-\infty}^{\infty}(b / 2)^{n}\left[1+\cosh \left(b^{n} x\right)\right]^{-1} .
$$

Then

$$
\begin{aligned}
\lim _{x \downarrow 0}|V(x)-U(x)| & =\lim _{x \downarrow 0} \sum_{n=-\infty}^{-1}(b / 2)^{n}\left[1+\cosh \left(b^{n} x\right)\right]^{-1} \\
& =\sum_{n=1}^{\infty}(2 / b)^{n}=((b / 2)-1)^{-1}=C_{0}
\end{aligned}
$$

On the other hand (here $k=n+1$ again)

We have

$$
\begin{aligned}
V(b x) & =\sum_{n=-\infty}^{\infty}(b / 2)^{n}\left[1+\cosh \left(b^{n+1} x\right)\right]^{-1} \\
& =(2 / b) \sum_{k=-\infty}^{\infty}(b / 2)^{k}\left[1+\cosh \left(b^{k} x\right)\right]^{-1}=\frac{2}{b} V(x) .
\end{aligned}
$$

$$
K(x)=V(x) x^{\gamma} ; \quad \gamma=1-\left(\log _{2} b\right)^{-1}
$$

and

$$
K(b x)=V(b x)(b x)^{\gamma}=\frac{2}{b} V(x) b^{\gamma} x^{\gamma}=K(x) .
$$

Reasoning analogous to the previous case implies analyticity of the function $K(x)$ defined by (31). It completes the proof of Lemma 8. It is noteworthy that the properties a) and b) of function $K(x)$ from Lemma 8 imply the estimate

$$
\left|K^{\prime}(x)\right| \leqq\left(\frac{C}{x}\right), \quad x>0 .
$$

Now when $b=2$ by (28) and (29) we have

$$
\chi_{2}=\left(T b^{2} / 4\right) B^{2}(h, T)\left[\frac{1}{2} \log _{2}(2|\Psi(h, T)|)^{-1}+K(2 \Psi(h, T))+r_{0}(h, T)\right],
$$

where $\lim _{h \downarrow 0} r_{0}(h, T)=0$ (here and below we indicate the dependence of all functions on $T$ again).

Using estimates from Lemma 7:

$$
|B(h, T)-A(T)(2 / b T)| \leqq C h^{2} ; \quad|\Psi(h, T)-A(T)(2|h| / b T)| \leqq C|h|^{3},
$$


finally, due to (32), we obtain the representation

$$
\chi_{2}=\frac{A^{2}(T)}{2 T}\left[\log _{2}(1 /|h|)+2 K\left(\frac{2 A(T)|h|}{T}\right)\right]+r_{1}(h, T) .
$$

When $b>2$ by (30) and (31) we have

$$
\chi_{2}=\left(T b^{2} / 4\right) B^{2}(h, T)\left[|b \Psi(h, T)|^{-\gamma} K(b \Psi(h, T))+\bar{r}_{0}(h, T)\right],
$$

where $\lim _{h \downarrow 0} \bar{r}_{0}(h, T)=C_{0},\left|C_{0}\right|<\infty$.

Analogously as in (33) we obtain

$$
\chi_{2}=\frac{A^{2}(T)}{T}\left[\frac{2 A(T)|h|}{T}\right]^{-\gamma} K\left(\frac{2 A(T)|h|}{T}\right)+\bar{r}_{1}(h, T) .
$$

Since $\left|\chi_{2}-\chi_{1}\right|$ has a limit when $h \downarrow 0$, formulae (33) and (34) are equivalent to the asymptotics of the susceptibility formulated in Proposition 1. The properties of function $K$ are established in Lemma 8. Thus proof of Proposition 1 is completed.

\section{Appendix B}

To prove Proposition 2 we will use another variant of the RG variables, namely $\xi_{n}$ $=\sqrt{t_{n} z_{n}}=\left(Q_{n} / P_{n}\right)^{1 / b}$ and $z_{n}$. Then from (10) it follows

$$
F=-\frac{J}{2}-h-\frac{T}{2} \sum_{n=0}^{\infty}(2 b)^{-n} \ln \left(1+\xi_{n}^{2 b}\right) .
$$

Sequence $\left\{\left(\xi_{n}, z_{n}\right), n=0,1, \ldots\right\}$ satisfies recurrent equations:

$$
\begin{gathered}
\xi_{n+1}=\left(\frac{Q_{n+1}}{P_{n+1}}\right)^{1 / b}=\frac{\left(Q_{n} / P_{n}\right)\left(1+\left(R_{n} / P_{n}\right)\right)}{1+\left(Q_{n}^{2} / P_{n}^{2}\right)}=\frac{\xi_{n}^{b}\left(1+z_{n}^{b}\right)}{1+\xi_{n}^{2 b}} \\
z_{n+1}=\left(\frac{R_{n+1}}{P_{n+1}}\right)^{1 / b}=\frac{R_{n}^{2}+Q_{n}^{2}}{P_{n}^{2}+Q_{n}^{2}}=\frac{z_{n}^{2 b}+\xi_{n}^{2 b}}{1+\xi_{n}^{2 b}}
\end{gathered}
$$

with the initial conditions $\xi_{0}=\exp (-(h+J) / b T), z_{0}=\exp (-2 h / b T)$. Note that

$$
\xi_{n+1}=\frac{2 \xi_{n}^{b}}{1+\xi_{n}^{2 b}} \equiv g\left(\xi_{n}\right),
$$

when $z_{n}=1$. Let $\xi_{c}=\sqrt{t_{c}}$ be the fixed point of the map $\xi \rightarrow g(\xi)$. If $\xi_{0}<\xi_{c}$, then iterations $\xi_{n+1}=g\left(\xi_{n}\right)$ go to zero and there exist constants $C_{0}=C_{0}\left(\xi_{0}, b\right)>0$, $C=C\left(\xi_{0}, b\right)>0$ such that

$$
0<\xi_{n}<C_{0} \exp \left(-C b^{n}\right) .
$$

(The function $g(\xi)$ has zero of the order $b$ at the point $\xi=0$.) If $z_{n} \leqq 1$, then by Eq. (36) $\xi_{n+1} \leqq g\left(\xi_{n}\right)$, so for $\xi_{0}<\xi_{c}$, (38) can be shown analogously as in Lemma 2.

By induction in $n$ in the same way as in Appendix $A$ it can be proved that

$$
\begin{gathered}
\left|\frac{\partial^{i} \xi_{n}}{\partial h^{i}}\right| \leqq C_{0}^{(i)} \exp \left(-C^{(i)} b^{n}\right), \\
\left|\frac{\partial^{i} z_{n}}{\partial h^{i}}\right| \leqq C_{1}^{(i)} \kappa_{i}^{n},
\end{gathered}
$$


where $i=1,2, \ldots, \kappa_{i}>0$. Hence it follows that the susceptibility $\chi(h, T)$ given by the series

$$
\chi=(T / 2) \sum_{n=0}^{\infty}(2 b)^{-n} \frac{\partial^{2}}{\partial h^{2}} \ln \left(1+\xi_{n}^{2 b}\right)
$$

converges uniformly in $h \geqq 0$, and consequently there exists $\lim _{h \downarrow 0} \chi(h, T)=\chi(T)$. This completes the proof of Proposition 2.

From estimates (39) the existence of all derivatives of the free energy at $h=+0$ when $T \leqq T_{c}$ follows directly.

On the other hand estimates obtained for quantities $\partial v_{n} / \partial h$ and $\partial \eta_{n} / \partial h$ in Appendix A enable us to prove the existence of the limit of the spontaneous magnetization $M(T)=\lim _{h \rightarrow+0} M(h, T)$, when $T>T_{c}$.

We have

$$
M(h, T)=-\frac{\partial F}{\partial h}=1-(b T / 2) \sum_{n=0}^{\infty}(2 b)^{-n}\left[1+\exp \left(b \eta_{n}+b v_{n}\right)\right]^{-1}\left(\frac{\partial \eta_{n}}{\partial h}+\frac{\partial v_{n}}{\partial h}\right) .
$$

According to Lemma 5

$$
\left|\frac{\partial \eta_{n}}{\partial h}\right| \leqq C_{0} b^{n} A_{n} \leqq C_{1} b^{n} ; \quad\left|\frac{\partial v_{n}}{\partial h}\right| \leqq C_{0} \exp \left(-C 2^{n}\right),
$$

hence the series in the formula of the magnetization converges uniformly when $h \rightarrow+0$ and $\lim _{h \rightarrow+0} M(h, T)=M(T)$ exists. A posteriori it means that $M(T)=0$, when $T>T_{c}$ (see Sect. 3 above).

\section{Appendix C}

To find a convenient representation for computations of transformation $\mathscr{R}^{-1}$ let us rewrite Eqs. (3) in the form

$$
\begin{gathered}
z_{n+1}=\frac{z_{n}^{b}+t_{n}^{b}}{z_{n}^{-b}+t_{n}^{b}}=\frac{z_{n}^{2 b}+t_{n}^{b} z_{n}^{b}}{1+z_{n}^{b} t_{n}^{b}} \\
t_{n+1}=\frac{z_{n}^{b}+z_{n}^{-b}+2}{z_{n}^{b}+z_{n}^{-b}+t_{n}^{b}+t_{n}^{-b}}=\frac{z_{n}^{b} t_{n}^{b}\left(1+z_{n}^{b}\right)^{2}}{\left(z_{n}^{2 b}+z_{n}^{b} t_{n}^{b}\right)\left(1+z_{n}^{b} t_{n}^{b}\right)} .
\end{gathered}
$$

Now using the substitution

$$
z_{n}^{b} t_{n}^{b}=u, \quad z_{n}^{b}=v
$$

we obtain

$$
z_{n+1}=\frac{v^{2}+u}{1+u}, \quad t_{n+1}=\frac{u(v+1)^{2}}{(u+1)\left(v^{2}+u\right)} .
$$

Let's solve these equations on $u$ and $v$. From the first one

$$
u=\frac{v^{2}-z_{n+1}}{z_{n+1}-1}
$$


and from the second equation (40)

$$
t_{n+1}=\frac{z_{n+1}-1}{z_{n+1}} \cdot \frac{v^{2}-z_{n+1}}{(v-1)^{2}}
$$

where $v \neq \pm 1$. So we have

$$
z_{n+1} t_{n+1}\left(v^{2}-2 v+1\right)=\left(z_{n+1}-1\right)\left(v^{2}-z_{n+1}\right),
$$

or the following quadratic equation for $v=z^{b}$ :

$$
v^{2}\left(z_{n+1} t_{n+1}-z_{n+1}+1\right)-2 z_{n+1} t_{n+1} v+\left(z_{n+1} t_{n+1}-z_{n+1}+z_{n+1}^{2}\right)=0 .
$$

Solving this equation we obtain $v$, and further from (41) we find $u$.

Acknowledgement. The authors express their gratitude to Prof. D. Surgailis for useful remarks.

\section{References}

1. Migdal, A.A.: Recurrence equations in gauge field theory. JETF 69, 810-822, 1457-1467 (1975)

2. Kadanoff, L.P.: Notes on Migdal's recursion formulae. Ann. Phys. 100, 359-394 (1976)

3. Berker, A.N., Ostlund, S.: Renormalization group calculations of finite systems. J. Phys. C. 12, 4961-4975 (1979)

4. Bleher, P.M., Žalys, E.: Existence of long-range order in the Migdal recursion equations. Commun. Math. Phys. 67, 17-42 (1979)

5. Griffiths, R.B., Kaufman, M.: Spin systems on hierarchical lattices. Introduction and thermodynamic limit. Phys. Rev. B 26, 5022-5039 (1982)

6. Griffiths, R.B., Kaufman, M.: Spin systems on hierarchical lattices. Some examples of soluble models. Phys. Rev. B 30, 244-249 (1984)

7. Kaufman, M., Griffiths, R.B.: First-order transitions in defect structures at a second-order critical point for the Potts model on hierarchical lattices. Phys. Rev. B 26, $5282-5284$ (1982)

8. Kaufman, M., Kardar, M.: Pseudodimensional variation and tricriticality of Potts models. Phys. Rev. B 30, 1609-1611 (1984)

9. Derrida, B., De Seze, L., Itzykson, C.: Fractal structure of zeros in hierarchical models. J. Stat. Phys. 30, 559-570 (1983)

10. Derrida, B., Itzykson, C., Luck, J.M.: Oscillatory critical amplitudes in hierarchical models. Commun. Math. Phys. 94, 115-127 (1984)

11. Kaufman, M.: Duality and Potts critical amplitudes on a class of hierarchical lattices. Phys. Rev. B 30, $413-414$ (1984)

12. Kaufman, M., Griffiths, R.B.: Infinite susceptibility at high temperatures in the MigdalKadanoff scheme. Phys. A 15, L 239-L 242 (1982)

13. Derrida, B., Eckmann, J.-P., Erzan, T.: Renormalization groups with periodic and aperiodic orbits. J. Phys. A 16, 893-906 (1983)

14. Bleher, P.M. Žalys, E.: Limit Gibbs distributions for Ising model on the hierarchical lattices. Lith. Math. J. 28, (1988) (in Russian)

15. Ruelle, D.: Statistical mechanics. Rigorous results. New York, Amsterdam: Benjamin 1969

Communicated by Ya. G. Sinai

Received April 15, 1988 